\title{
The extremely halotolerant black yeast Hortaea werneckii - a model for intraspecific hybridization in clonal fungi
}

\author{
Polona Zalar ${ }^{1 *}$ (D), Jerneja Zupančič ${ }^{1}$, Cene Gostinčar ${ }^{1,2}$, Janja Zajc ${ }^{3}$, G. Sybren de Hoog ${ }^{4,5}$, Filomena De Leo ${ }^{6}$,
} Armando Azua-Bustos ${ }^{7,8}$ and Nina Gunde-Cimerman ${ }^{1}$

\begin{abstract}
The polymorphic black yeast Hortaea werneckii (Capnodiales, Ascomycota) is extremely halotolerant (growth from 0 to $30 \%[\mathrm{w} / \mathrm{v}] \mathrm{NaCl}$ ) and has been extensively studied as a model for halotolerance in Eukaryotes for over two decades. Its most frequent sources are hypersaline environments and adjacent sea-water habitats in temperate, subtropical and tropical climates. Although typically saprobic, H. werneckii can also act as a commensal coloniser on human skin, causing tinea nigra on hands and soles. Here, we report that addition of $\mathrm{NaCl}$ to culture media expands the growth range of $\mathrm{H}$. werneckii to $37^{\circ} \mathrm{C}$, which explains its colonisation of human skin, with its increased salinity. The morphological and physiological plasticity/ versatility of $H$. werneckii indicate that a species complex might be involved. This was investigated in this polyphasic taxonomic analysis based on the global diversity of $H$. werneckii strains collected from hypersaline environments, and from humans and animals. Analysis of D1/ D2domains of 285 and internal transcribed spacer rDNA revealed 10 and 17 genotypes, respectively, that were not always compliant. The genotypes have global distributions. Human and environmental strains with the same genotypes are intermingled. Due to the limited number of phylogenetically informative characters in the ribosomal DNA dataset, the partial genes encoding for $\beta$-tubulin (BTB) and mini-chromosome maintenance protein (MCM7) were also sequenced. The use of these genes was hampered by ambiguous sequences obtained by Sanger sequencing, as a consequence of the diploid and highly heterozygous genome of many $H$. werneckil strains. Analysis of the BTB and MCM7 genes showed that in some cases two copies of the gene from the same genome are positioned in distant phylogenetic clusters of the intraspecific gene tree. Analysis of whole-genome sequences of selected $H$. werneckii strains generally confirmed the phylogenetic distances estimated on the basis of ribosomal genes, but also showed substantial reticulation within the phylogenetic history of the strains. This is in line with the hypothesis that the diploid genomes of $\mathrm{H}$. werneckii were formed by hybridizations, which have sometimes occurred between relatively divergent strains.
\end{abstract}

Keywords: Morphology, Clustering, NaCl tolerant enyzmes, ITS, D1/D2 rDNA, MCM7, BTB, Genomes

\section{INTRODUCTION}

The ascomycetous black yeast Hortaea werneckii (Capnodiales, Teratosphaeriaceae) is the most extremely halotolerant fungus known, and as such, it has become an important model organism for the study of halotolerance in Eukarya (Plemenitaš et al. 2008, 2014, Gunde-Cimerman et al. 2018). Its primary ecological niche are natural hypersaline

\footnotetext{
* Correspondence: polona.zalar@bf.uni-lj.si

${ }^{1}$ Department of Biology, Biotechnical Faculty, University of Ljubljana, Večna pot 111, SI-1000 Ljubljana, Slovenia

Full list of author information is available at the end of the article
}

environments worldwide. It has been isolated from thalasso-haline brines in salterns on three continents, with numerous locations ranging from temperate (Mediterranean) (Gunde-Cimerman et al. 2000, Cabañes et al. 2012, Gunde-Cimerman \& Zalar 2014, Elsayed et al. 2016) to subtropical and tropical (Mexico, Puerto Rico) (Díaz-Muñoz \& Montalvo-Rodríguez 2005, Cantrell et al. 2006) climate zones, although never in subpolar and polar regions. Hortaea werneckii is more frequently found in eutrophic thalasso-haline waters at moderate temperatures, while it is not known to occur in oligotrophic or 
athalasso-haline hypersaline waters, and only rarely in hypersaline waters with elevated temperatures (Plemenitaš \& Gunde-Cimerman 2005). Occasionally, H. werneckii has been found in seawater (Iwatsu \& Udagawa 1988), on rocks adjacent to seawater (Ruibal et al. 2009), and inhabiting sea sponges (Brauers et al. 2001), corals (Amend et al. 2012, Xu et al. 2014), marine fish (Todaro et al. 1983), salted freshwater fish (Mok et al. 1981), beach soil (de Hoog \& Guého 1998), saltern microbial mats (Cantrell et al. 2006), and salt marsh plants (Formoso et al. 2015), and as an endophyte in mangrove plants (Chen et al. 2012). Recently, it was discovered in an indoor dust sample collected in Hawaii (Humphries et al. 2017). In agreement with the extremotolerant nature, it was isolated from 2500 $m$ in depth in the Mediterranean Sea (De Leo et al. 2018), from sediments $5000 \mathrm{~m}$ below sea level in the Central Indian Basin (Singh et al. 2012), and in sediments $4000 \mathrm{~m}$ below sea level in the East India Ocean (Zhang et al. 2014). Using internal transcribed spacer (ITS) clone libraries, $H$. werneckii operational taxonomic units were detected in the South China Sea in methane-hydrate-bearing deep-sea sediments (Lai et al. 2007). In spite of the wide distribution in marine environments, brines in solar salterns are considered the primary ecological niche, where at salinities above $20 \%(\mathrm{w} / \mathrm{v}) \mathrm{NaCl}, H$. werneckii represents from 70 to $80 \%$ of all fungal isolates (Gunde-Cimerman et al. 2000; Butinar et al. 2005), with densities of up to $1400 \mathrm{CFU} / \mathrm{L}$.

Hortaea werneckii is the only fungus that can grow across almost the whole range of $\mathrm{NaCl}$ concentrations, from 0 to $30 \% \mathrm{NaCl}$, with a broad optimum between 6 and $14 \% \mathrm{NaCl}$ (Butinar et al. 2005). As well as $\mathrm{NaCl}, H$. werneckii can also tolerate up to $2 \mathrm{M}$ concentrations of the chaotropic salt $\mathrm{MgCl}_{2}$, and up to $1.7 \mathrm{M} \mathrm{CaCl}_{2}$, and it has been isolated from bitterns, i.e. $\mathrm{MgCl}_{2}$-rich leftover brines after harvesting of $\mathrm{NaCl}$ (Zajc et al. 2014). The species also grows on osmotic media with $10 \% \mathrm{NaCl}$ with $12 \%$ glucose (e.g. malt extract, yeast extract, $10 \%$ $\mathrm{NaCl}, 12 \%$ glucose agar) and with $50 \%$ glucose (e.g., malt extract, yeast extract, 50\% glucose agar) (Gunde-Cimerman et al. 2000, Humphries et al. 2017).

Until 2000, H. werneckii was primarily known as the aetiological agent of a skin disorder known as tinea nigra, a superficial infection of the human hands and feet that can occur in warmer climates (de Hoog et al. 2000, Perez et al. 2005, Bonifaz et al. 2008). Infections have been associated with superficially abraded skin exposed to hypersaline tidal waters (de Hoog \& Gerrits van der Ende 1992) in combination with hyperhydrosis (Bonifaz et al. 2008, Paneque Rodríguez et al. 2015). The species has been associated with animal bites (Rossetto et al. 2014). The pathogenic potential of $H$. werneckii has, however, remained unclear, as it was generally considered that the species could not grow at $37{ }^{\circ} \mathrm{C}$ (de Hoog et al. 2000, Ng et al. 2005).
Under extremely saline conditions, $H$. werneckii has an extremophilic ecotype, which is characterised by enhanced melanization, changes in cell size and meristematic growth, and changes in colony appearance (Kogej et al. 2007). At the molecular level, the responses of $H$. werneckii to high concentrations of toxic sodium ions have involved changes in the expression of genes associated with the synthesis of compatible solutes, regulation of intracellular alkali-metal cation concentrations, modifications of cell membrane lipid composition, and changes in cell-wall ultrastructure and morphology (Plemenitaš et al. 2014). Due to these adaptations, $H$. werneckii has an outstanding ability to overcome the turgor loss and sodium toxicity that are typical of hypersaline environments (Gostinčar et al. 2011).

Genome sequencing of the $H$. werneckii strain EXF-2000 (Lenassi et al. 2013, Sinha et al. 2017) has revealed that with almost $50 \mathrm{Mb}$, the $H$. werneckii genome is larger than the genomes of other sequenced representatives of Capnodiales. The $H$. werneckii genome contained close to 16,000 genes, of which $90 \%$ were present in two copies, with an average of 5\% amino-acid sequence divergence between the predicted proteins. Genomic data fit well with the molecular studies on individual genes performed earlier, as all of the genes studied to this point were duplicated and coded for two functionally identical proteins (Vaupotič \& Plemenitaš 2007, Vaupotič et al. 2008).

Recent analyses of the whole genome sequences of 11 additional strains revealed that two were haploid and the remaining nine were diploid. Differences between the subgenomes of individual strains and their detailed phylogenetic analyses have shown that the diploid genomes were most likely produced by hybridization between relatively divergent strains (Gostinčar et al. 2018). At the same time, apart from identification of a heterothallic mating locus, the comparative genomic analyses have failed to find any traits that can be linked to sexual reproduction.

In summary, the global presence of $H$. werneckii in hypersaline environments, its role as the most important model for halotolerance in Eukarya, its extensive physiological plasticity and adaptability, the availability of numerous strains collected from various osmotic environments, and its particular combination of hybridization with an exclusively or primarily clonal reproduction mode (as revealed by comparative genomics) make $H$. werneckii an attractive model organism.

The present study compared 98 strains, of which a selection was subjected to classical taxonomic analyses (macro-, microscopy, assimilation tests), and was tested for halotolerance and thermotolerance and the production of selected pathogenicity-related enzymes (proteases, esterases), both with and without the addition of 
salt. Taxonomic analyses have been supported by comparative analyses of the most frequently used barcodes, ITS and D1/D2 28S rDNA. Indeed, apart from the ribosomal regions, almost all of the other genes were present in two different copies per strain, which prevented classical taxonomic phylogenetic approaches based on analyses of household genes. Therefore, in addition to ambiguous Sanger sequences and adjusted algorithms to work with such sequences, sequences derived from whole-genome sequencing of 11 strains were also considered. The taxonomic study presented was aimed at definition of the variability in $H$. werneckii, the description of potentially new species, and the definition of genotypes that might correlate to particular ecological habitats.

\section{METHODS}

This study presents a taxonomic analysis of 98 strains (Table 1) that were mostly isolated from brine or bitterns of three Mediterranean salterns (47 strains), adjacent sea and deep sea environments (10 strains), and air above hypersaline ponds (1). Five strains were also included from patients living in the Mediterranean area (i.e., Italy, Portugal, France), as well as strains from animals (7) and plants (3). One strain was food-borne (it formed a black biofilm on cheese brine), one originated from the air in Japan, and one was of unknown source. Twenty-two strains originated from a coastal cave located in the Coastal Range of the Atacama Desert (Chile). These strains were isolated from spider webs attached to the cave wall, from a newly described species of microalga (Dunaliella atacamensis; Azúa-Bustos et al. 2010), and directly from the cave walls and from sand underneath the spider webs.

\section{Isolation of strains and sampling sites}

The Hortaea werneckii strains studied are listed in Table 1, and were collected over a 20-year period. They are maintained in the Microbial Culture Collection Ex of the Infrastructural Centre Mycosmo, MRIC UL, Slovenia (http://www.ex-genebank.com), in the Department of Biology, Biotechnical Faculty, University of Ljubljana (Slovenia). Most of these strains originated from brine and bitterns of the Sečovlje seasonal solar salterns on the northern Adriatic coast (Zalar et al. 1999, GundeCimerman et al. 2000, Butinar et al. 2005), at the border between Slovenia and Croatia (sub-Mediterranean climate). Brines from salterns in Spain (i.e. Santa Pola and Ebro River Delta) were sampled in 1999 and 2000, and in Namibia (i.e. Skeleton coast) in 2003. Isolations were performed as described in Gunde-Cimerman et al. (2000), by filtration of water and placing the filters on hypersaline culture media. Strains from bitterns were isolated with additional enrichment in half-strength liquid media: malt extract $(\mathrm{pH} 7,3.5)$, yeast nitrogen base (YNB), and corn steep liquid supplemented with $50 \mathrm{mg} / \mathrm{L}$ chloramphenicol. Enrichments were carried out in $500 \mathrm{~mL}$ Erlenmeyer flasks with $50 \mathrm{~mL}$ medium and $50 \mathrm{~mL}$ original bittern. After $7 \mathrm{~d}$ incubation on a rotary shaker at $25^{\circ} \mathrm{C}$ at $180 \mathrm{rpm}, 100 \mu \mathrm{L}$ of the broth was cultured on DG18, malt extract agar (MEA) $+3 \mathrm{M} \mathrm{NaCl}$ and MEA + 1.5 $\mathrm{M} \mathrm{MgCl}_{2}$.

The strains from Puerto Rico salterns originate from the study of Cantrell et al. (2006). Seawater and deep sea strains were collected in 2013 during the DEEP-PRESSURE Cruise on board the R/V Urania (Smedile et al. 2015), from water columns $25-2500 \mathrm{~m}$ in depth, sampled at four stations in the Mediterranean Sea: Station Vector in the Tyrrhenian Sea $\left(39^{\circ} 32^{\prime} 00.6^{\prime \prime} \mathrm{N} 13^{\circ} 22^{\prime} 28.5^{\prime \prime}\right.$ E, Station KM3 in the Ionian Sea $\left(36^{\circ} 30^{\prime} 61.3^{\prime \prime} \mathrm{N} 15^{\circ} 40^{\prime}\right.$ $\left.59.9^{\prime \prime} \mathrm{E}\right)$, and above the anoxic hypersaline lakes L'Atalante $\left(35^{\circ} 18^{\prime} 92^{\prime \prime} \mathrm{N} 21^{\circ} 23^{\prime} 92^{\prime \prime} \mathrm{E}\right)$ and Medee $\left(34^{\circ} 24^{\prime} 00^{\prime \prime} \mathrm{N}\right.$ $22^{\circ} 26^{\prime} 99^{\prime \prime} \mathrm{E}$ ), in the central and south Mediterranean Sea, respectively (de Leo et al. 2018). Strains from corals in the Pacific Ocean (China) were isolated by Luo et al. (2017). A large number of strains were collected in 2009 and 2017 by Azúa-Bustos from soil, sand and spider webs collected inside Huanillos cave, which is located under an ancient guano deposit that is rich in ammonium nitrate, urate and phosphates. This cave is in the Coastal Range hills of the Atacama Desert, south of the city of Iquique, Chile (Azúa-Bustos et al. 2010), as also described by Martinelli et al. (2017).

All of the strains were single colony isolates after plate streaking. All of the human-, animal-, and plant-related strains were obtained from the Westerdijk Institute for Fungal Biodiversity culture collection (CBS; http://www. westerdijkinstitute.nl/Collections/).

\section{DNA extraction and sequencing}

DNA extraction was performed according to van den Ende \& de Hoog (1999). About 50 ng template DNA was used in the PCRs. The complete ITS and the D1/D2 domain of $28 \mathrm{~S}$ rDNA were amplified with the primers ITS4 and ITS5 (White et al. 1990) or NL1 and NL4 (O'Donnell 2000). A fragment of the $\beta$-tubulin gene $(B T B)$ was amplified with primers BT2a and BT2b (Glass \& Donaldson 1995), and a fragment of DNA replication licensing factor required for DNA replication initiation and cell proliferation (MCM7) was amplified with primers Mcm7-709for and Mcm7-1348rev (Schmitt et al. 2009). The same primers were also used in the sequencing reactions. Sequences were generated through Sanger sequencing (16 ABI 3730xl) using a commercial provider (Microsynth, Wien, Austria). The sequence chromatograms were manually checked for quality using the FinchTV software (Geospiza, Los Altos, CA) and assembled and edited using SeqMan 3.61 (DNAStar, 
Table 1 Hortaea werneckii strains treated in this study

\begin{tabular}{|c|c|c|c|c|c|}
\hline \multirow{2}{*}{$\begin{array}{l}\text { Strain n } \\
\text { EXF- }\end{array}$} & \multirow[b]{2}{*}{ Other collections } & \multirow[t]{2}{*}{ ORIGIN (sample, collection date, location) } & \multirow[b]{2}{*}{$\begin{array}{l}\text { Genotype } \\
\text { (28S/ITS) }\end{array}$} & \multicolumn{2}{|c|}{ GenBank acc. No. } \\
\hline & & & & D1/D2 28S rDNA & ITS rDNA \\
\hline \multicolumn{6}{|c|}{ Hypersaline brine and bittern in salterns } \\
\hline 9 & & brine, 11/1998, Salinas de la Trinitat (Ebre Delta), Spain & $\mathrm{D} / 8$ & MH327587 & MH327684 \\
\hline 64 & & brine, 7/1998, Salinas de la Trinitat (Ebre Delta), Spain & $B / 7$ & MH327569 & MH327666 \\
\hline 96 & & brine, 11/1998, Salinas de la Trinitat, Spain & $\mathrm{D} / 8$ & MH327585 & MH327682 \\
\hline 120 & & brine, pond 3, 11/1998, Salinas Santa Pola, Spain & $B / 7$ & MH327575 & MH327672 \\
\hline 132 & & brine, pond 5, 11/1998, Salinas Santa Pola, Spain & $B / 7$ & MH327576 & MH327673 \\
\hline 152 & & brine, pond 1, 9/1996, Sečovlje salterns, Slovenia & $B / 7$ & MH327577 & MH327674 \\
\hline 153 & & brine, pond 1, 5/1996, Sečovlje, Slovenia & $B / 7$ & MH327597 & MH327694 \\
\hline 154 & & brine, pond 1, 9/1996, Sečovlje, Slovenia & $B / 7$ & MH327581 & MH327678 \\
\hline 225 & & brine, pond 1, 8/1996, Sečovlje, Slovenia & $B / 7$ & MH327568 & MH327665 \\
\hline 241 & & brine, pond 1, 6/1996, Sečovlje, Slovenia & $A / 1$ & MH327539 & MH327636 \\
\hline 247 & & brine, pond 1, 9/1996, Sečovlje, Slovenia & $A / 1$ & MH327541 & MH327638 \\
\hline 269 & & brine, 11/1998, Santa Pola, Spain & $B / 7$ & MH327579 & MH327676 \\
\hline 489 & & brine, pond 3, 11/1998, Salinas de la Trinitat, Spain & $\mathrm{C} / 9$ & MH327592 & MH327689 \\
\hline 537 & & brine, pond 1, 8/1996, Sečovlje, Slovenia & $B / 7$ & MH327571 & MH327668 \\
\hline 554 & & brine, pond 1, 8/1996, Sečovlje, Slovenia & $A / 1$ & MH327532 & MH327629 \\
\hline 561 & & brine, 3/2000, Salterns at the Skeleton coast (Atlantic), Namibia & $J / 13$ & MH327618 & MH327626 \\
\hline 562 & & brine, 3/2000, Salterns at the Skeleton coast (Atlantic), Namibia & $\mathrm{E} / 4$ & MH327563 & MH327660 \\
\hline 631 & & brine, 11/1998, Santa Pola, Spain & $\mathrm{D} / 8$ & MH327586 & MH327683 \\
\hline 647 & & brine, 11/1998, Santa Pola, Spain & $\mathrm{D} / 7$ & MH327564 & MH327661 \\
\hline 2516 & & brine, 12/2004, Candelaria, Puerto Rico & $1 / 11$ & MH327546 & MH327643 \\
\hline 2685 & CBS 100456 & brine, 1998, Sečovlje, Slovenia & $B / 7$ & MH327590 & MH327687 \\
\hline 2782 & & brine, pond 4, 8/1996, Sečovlje, Slovenia & $B / 7$ & MH327598 & MH327695 \\
\hline 2783 & & brine, pond 2, 8/1996, Sečovlje, Slovenia & $\mathrm{D} / 8$ & MH327596 & MH327693 \\
\hline 2785 & & brine, pond 1, 5/1996, Sečovlje, Slovenia & $\mathrm{C} / 9$ & MH327593 & MH327690 \\
\hline 2787 & & immersed wood in brine, pond 1, 1/1997, Sečovlje, Slovenia & $\mathrm{B} / 7$ & MH327580 & MH327677 \\
\hline 2788 & & brine, pond 2, 7/1996, Sečovlje, Slovenia & $\mathrm{D} / 8$ & MH327584 & MH327681 \\
\hline 3506 & PR 1105-73 & brine, Candelaria, Puerto Rico & $E / 5$ & MH327550 & MH327647 \\
\hline 3846 & PR08-Candel.12 & brine, 3/2008, Candelaria, Puerto Rico & $A / 16$ & MH327545 & MH327642 \\
\hline 4642 & & brine, pond 1, 9/1996, Sečovlje, Slovenia & $B / 7$ & MH327572 & MH327669 \\
\hline 4662 & & brine, pond 1, 9/1996, Sečovlje, Slovenia & $\mathrm{B} / 7$ & MH327573 & MH327670 \\
\hline 4667 & & brine, pond 1, 7/1996, Sečovlje, Slovenia & $B / 7$ & MH327574 & MH327671 \\
\hline 4717 & & brine, pond 1, 8/1996, Sečovlje, Slovenia & $\mathrm{B} / 10$ & MH327591 & MH327688 \\
\hline 7620 & & brine, reservoir, 12/2009, Sečovlje, Slovenia & $\mathrm{B} / 7$ & MH327578 & MH327675 \\
\hline 7637 & & brine, reservoir, 12/2009, Sečovlje, Slovenia & $\mathrm{B} / 7$ & MH327566 & MH327663 \\
\hline 7638 & & brine, reservoir 12/2009, Sečovlje, Slovenia & $\mathrm{B} / 7$ & MH327565 & MH327662 \\
\hline 10304 & & brine, 11/2015, Sečovlje salterns, Slovenia & $B / 7$ & MH327600 & MH327698 \\
\hline 10813 & & bittern, 11/2016, Sečovlje salterns, Slovenia & $B / 7$ & MH327601 & MH327699 \\
\hline 10820 & & bittern, 11/2016, Sečovlje salterns, Slovenia & $B / 7$ & MH327582 & MH327679 \\
\hline 10828 & & brine, 11/2016, Sečovlje salterns, Slovenia & $A / 1$ & MH327602 & MH327700 \\
\hline 10830 & & brine, 11/2016, Sečovlje salterns, Slovenia & $\mathrm{D} / 8$ & MH327588 & MH327685 \\
\hline 10831 & & brine, 11/2016, Sečovlje salterns, Slovenia & $\mathrm{B} / 8$ & MH327603 & MH327701 \\
\hline
\end{tabular}


Table 1 Hortaea werneckii strains treated in this study (Continued)

\begin{tabular}{|c|c|c|c|c|c|}
\hline \multirow{2}{*}{$\begin{array}{l}\text { Strain no. } \\
\text { EXF- }\end{array}$} & \multirow[b]{2}{*}{ Other collections } & \multirow[t]{2}{*}{ ORIGIN (sample, collection date, location) } & \multirow[b]{2}{*}{$\begin{array}{l}\text { Genotype } \\
\text { (28S/ITS) }\end{array}$} & \multicolumn{2}{|c|}{ GenBank acc. No. } \\
\hline & & & & D1/D2 285 rDNA & ITS rDNA \\
\hline 10834 & & brine, 11/2016, Sečovlje salterns, Slovenia & $B / 7$ & MH327604 & MH327702 \\
\hline 10842 & & brine, 11/2016, Sečovlje salterns, Slovenia & $\mathrm{C} / \mathrm{9}$ & MH327595 & MH327692 \\
\hline 10843 & & brine, 11/2016, Sečovlje salterns, Slovenia & $\mathrm{B} / 9$ & MH327605 & MH327703 \\
\hline 10957 & & bittern, 11/2016, Sečovlje salterns, Slovenia & $B / 7$ & MH327607 & MH327705 \\
\hline 10974 & & brine, 11/2016, Sečovlje salterns, Slovenia & $\mathrm{C} / 9$ & MH327608 & MH327706 \\
\hline 10975 & & brine, 11/2016, Sečovlje salterns, Slovenia & $\mathrm{C} / \mathrm{9}$ & MH327609 & MH327707 \\
\hline \multicolumn{6}{|c|}{ Sea water and related habitats } \\
\hline 166 & CBS 100496 & sea water-sprayed marble, Delos, Greece & $A / 1$ & MH327538 & MH327635 \\
\hline 2684 & & sea water, 1998, Slovenia & $\mathrm{C} / 9$ & MH327594 & MH327691 \\
\hline 2686 & CBS 373.92 & beach sand, 1992, La Palma, Spain & $\mathrm{H} / 1$ & MH327530 & MH327627 \\
\hline 10508 & AT $25 f$ & Mediterranean sea, depth 25 m, 12/2013, "Atalante" station & $E / 4$ & MH327555 & MH327652 \\
\hline 10509 & KM3 200r & Mediterranean sea, depth 200 m, 12/2013, "KM3" station & $E / 4$ & MH327556 & MH327653 \\
\hline 10510 & M 94 & Mediterranean sea, depth 94 m, 12/2013, "Medee" station & $E / 4$ & MH327557 & MH327654 \\
\hline 10511 & $\vee 25 a$ & Mediterranean sea, depth 25 m, 12/2013, "Vector" station & $E / 4$ & MH327558 & MH327655 \\
\hline 10512 & $\vee 25 c$ & Mediterranean sea, depth 25 m, 12/2013, "Vector" station & $E / 4$ & MH327559 & MH327656 \\
\hline 10513 & $\vee 2500 b$ & Mediterranean sea, depth 2500 m, 12/2013, "Vector" station & $E / 4$ & MH327560 & MH327657 \\
\hline 10853 & no. 445345 & beach sand, Portugal & $B / 7$ & MH327606 & MH327704 \\
\hline \multicolumn{6}{|c|}{ Animal related strains } \\
\hline 156 & CBS 116.90 & fish kantar (Spondyliosoma cantharus), eye infection, unknown & $B / 7$ & MH327570 & MH327667 \\
\hline 157 & CBS 115.90 & frog, kidney, Brazil & $E / 4$ & MH327554 & MH327651 \\
\hline 2683 & CBS 117.90 & fish Osteoglossum bicirrhosum, Brazil & $A / 1$ & MH327542 & MH327639 \\
\hline 4625 & CBS 100455 & red coral (Corallium rubrum), 8/1996, Ugljan, Croatia & $B / 7$ & MH327567 & MH327664 \\
\hline 12619 & MCCC 3A00558 & corals, Pacific Ocean, China & $A / 5$ & MH327619 & MH327714 \\
\hline 12620 & MCCC 3A00680 & corals, Pacific Ocean, China & $E / 15$ & MH327620 & MH327716 \\
\hline 12684 & MCCC 3A00555 & corals, Pacific Ocean, China & $A / 5$ & MH327621 & MH327715 \\
\hline \multicolumn{6}{|c|}{ Plant related strains } \\
\hline 161 & CBS 706.76 & unknown tree leaf, Senegal & $\mathrm{D} / 8$ & MH327583 & MH327680 \\
\hline 2688 & CBS 255.96 & Casuarina equisetifolia, Canarian Islands, Spain & $B / 12$ & MH327589 & MH327686 \\
\hline 2690 & CBS 707.76 & sooty mould, Sri Lanca & $F / 4$ & MH327552 & MH327649 \\
\hline \multicolumn{6}{|c|}{ Human related strains } \\
\hline 151 & CBS $107.67^{\top}$ & man, tinea nigra, Portugal & $\mathrm{G} / 1$ & MH327544 & MH327641 \\
\hline 155 & CBS 359.66 & man, unknown & $A / 1$ & MH327543 & MH327640 \\
\hline 171 & CBS 111.31 & man, keratomycosys, Brazil & $E / 4$ & MH327551 & MH327648 \\
\hline 177 & CBS 705.76 & man, tinea nigra, France & $A / 1$ & MH327540 & MH327637 \\
\hline 2682 & CBS 126.35 & man, trichomycosys nigra, 9/1935, Italy & $F / 6$ & MH327561 & MH327658 \\
\hline \multicolumn{6}{|c|}{ Strains from arid environments } \\
\hline 6651 & & spiderweb with (w) algae, 1/2010, Huanillos cave, Atacama, Chile & $A / 1$ & MH327533 & MH327630 \\
\hline 6652 & & spiderweb w algae, 1/2010, Huanillos cave, Atacama, Chile & $A / 1$ & MH327534 & MH327631 \\
\hline 6653 & & spiderweb w algae, 1/2010, Huanillos cave, Atacama, Chile & $A / 1$ & MH327535 & MH327632 \\
\hline 6654 & & spiderweb without (wo) algae, 1/2010, Huanillos cave, Atacama, Chile & $A / 3$ & MH327549 & MH327646 \\
\hline 6655 & & spiderweb wo algae, 1/2010, Huanillos cave, Atacama, Chile & $A / 1$ & MH327536 & MH327633 \\
\hline 6656 & & rock, 1/2010, Huanillos cave, Atacama, Chile & $\mathrm{A} / 2$ & MH327547 & MH327644 \\
\hline
\end{tabular}


Table 1 Hortaea werneckii strains treated in this study (Continued)

\begin{tabular}{|c|c|c|c|c|c|}
\hline \multirow{2}{*}{$\begin{array}{l}\text { Strain no. } \\
\text { EXF- }\end{array}$} & \multirow[b]{2}{*}{ Other collections } & \multirow[t]{2}{*}{ ORIGIN (sample, collection date, location) } & \multirow[b]{2}{*}{$\begin{array}{l}\text { Genotype } \\
\text { (28S/ITS) }\end{array}$} & \multicolumn{2}{|c|}{ GenBank acc. No. } \\
\hline & & & & D1/D2 285 rDNA & ITS rDNA \\
\hline 6658 & & spiderweb wo algae, 1/2010, Huanillos cave, Atacama, Chile & $\mathrm{A} / 1$ & / & $\mathrm{MH} 327722$ \\
\hline 6663 & & spiderweb w algae, 1/2010, Huanillos cave, Atacama, Chile & $\mathrm{A} / 2$ & MH327622 & MH327718 \\
\hline 6664 & & spiderweb w algae, 1/2010, Huanillos cave, Atacama, Chile & $A / 1$ & MH327531 & MH327628 \\
\hline 6665 & & spiderweb w algae, 1/2010, Huanillos cave, Atacama, Chile & $A / 3$ & MH327548 & MH327645 \\
\hline 6666 & & spiderweb w algae, 1/2010, Huanillos cave, Atacama, Chile & $\mathrm{A} / 2$ & MH327623 & MH327719 \\
\hline 6667 & & spiderweb w algae, 1/2010, Huanillos cave, Atacama, Chile & $A / 17$ & MH327625 & MH327723 \\
\hline 6668 & & spiderweb w algae, 1/2010, Huanillos cave, Atacama, Chile & $\mathrm{A} / 2$ & MH327624 & MH327720 \\
\hline 6669 & & spiderweb w algae, 1/2010, Huanillos cave, Atacama, Chile & $A / 1$ & MH327537 & MH327634 \\
\hline 11528 & & sand, 2/2017, Huanillos cave, Atacama, Chile & $A / 1$ & MH327610 & MH327721 \\
\hline 11531 & & sand, 2/2017, Huanillos cave, Atacama, Chile & $\mathrm{A} / 2$ & MH327611 & MH327708 \\
\hline 11537 & & sand, 2/2017, Huanillos cave, Atacama, Chile & $A / 14$ & MH327612 & MH327709 \\
\hline 11538 & & sand, 2/2017, Huanillos cave, Atacama, Chile & $\mathrm{A} / 2$ & MH327613 & MH327710 \\
\hline 11539 & & sand, 2/2017, Huanillos cave, Atacama, Chile & $\mathrm{A} / 2$ & MH327614 & MH327711 \\
\hline 11540 & & sand, 2/2017, Huanillos cave, Atacama, Chile & $\mathrm{A} / 2$ & MH327615 & $\mathrm{MH} 327712$ \\
\hline 11547 & & sand, 2/2017, Huanillos cave, Atacama, Chile & $\mathrm{A} / 2$ & MH327616 & / \\
\hline 11548 & & sand, 2/2017, Huanillos cave, Atacama, Chile & $A / 1$ & MH327617 & MH327713 \\
\hline 2687 & CBS 410.51 & air, 2/1951, Japan & $\mathrm{F} / 4$ & MH327553 & MH327650 \\
\hline 4493 & & air, 1993, abandoned salterns Sečovlje, Slovenia & $\mathrm{nd} / 10$ & / & MH327696 \\
\hline \multicolumn{6}{|l|}{ Other } \\
\hline 4661 & CBS 122.32 & unknown & $F / 6$ & MH327562 & MH327659 \\
\hline 8422 & & biofilm from salted water of a cheese factory, Slovenia & $\mathrm{B} / 7$ & MH327599 & MH327697 \\
\hline
\end{tabular}

Madison, QI). PCR products that resulted in Sanger-derived BTB sequences that showed ambiguous signals were first purified from $1 \%$ agarose gels with GeneJET Gel extraction kits (ThermoFisher Scientific, Waltham, MA), and then cloned into the pJET vector and transformed into XL1Blue Escerichia coli using TransformAid bacterial transformation kits (ThermoFisher Scientific). Transformants were selected after overnight growth on Luria-Bertani agar medium supplemented with ampicillin. Plasmids were extracted from 20 transformant colonies using GeneJET plasmid miniprep kits (ThermoFisher Scientific) and the cloned $B T B$ fragments were sequenced as described above. The DNA sequence data have been deposited with GenBank (Table 1).

\section{ITS and 28S rDNA genotypes and genome variants}

ClustalW aligned ITS and 28S rDNA Sanger sequences were checked for single nucleotide polymorphisms and overall similarities using the unweighted pair group method with arithmetic averages (UPGMA) for cluster analyses. MEGA 5.2 was also used to select the nucleotide substitution model used in the UPGMA runs. Other settings included the use of gaps or missing data as partial deletions with a site coverage cut-off of 95\%, with nearest-neighbour-interchange used as a heuristic method. Internal branch support was assessed on the basis of 1000 bootstrapped datasets. Genotypes encountered in the ITS were numbered 1-17, and those for the $28 \mathrm{~S}$ rDNA A-J. Genotypes that were based on both loci were characterised by using first the letter code for the $28 \mathrm{rDNA}$ followed by a slash and then the number code of the ITS. The ITS (28S rDNA) alignment started with ATCATTA (ACGGCGA).

Tandem repeated ITS and 28S rDNA fragments from whole genome sequences were aligned with the ITS and $28 \mathrm{~S}$ rDNA reference sequences from strain EXF-2000 (= EXF-225) with BWA-MEM (Li \& Durbin 2009). Variant calling was performed with Samtools 1.5 ( $\mathrm{Li}$ et al. 2009) in the diploid mode, after the sequences were sorted and deduplicated.

\section{Phylogenetic analyses based on protein-encoding genes and whole genomes}

The two protein-encoding genes were separately analysed phylogenetically after their sequences were aligned with MAFFT, in automatic mode (Katoh \& Toh 2008). Sanger sequences of $B T B$ not showing ambiguous bases, which included those with clean chromatograms 
obtained after cloning, were aligned with $B T B$ fragments obtained from whole genomes. Alignments of the $M C M 7$ sequences were based on sequences without ambiguous signals and also with those showing ambiguous signals, from which the estimated haplotypes were generated using PHASE 2.1 (Stephens et al. 2001), using 10 haplotype pairs from whole genome sequences as known phases (Gostinčar et al. 2018). The resulting haplotype pairs that showed identical tree positions in preliminary phylogenetic analyses were collapsed into one sequence. The MCM7 alignments also included sequences derived from sequenced genomes. Best suiting substitution models were determined using jModelTest 2.1.10 (Darriba et al. 2012) and phylogenetic trees were generated using PhyML 3.1 (Guindon et al. 2010). The alpha parameter of the gamma distribution of substitution rate categories and the proportion of invariable sites were estimated using PhyML. Branch supports were estimated using aLRT, as Chi2-based supports.

A whole genome phylogenetic network was calculated as described by Gostinčar et al. (2018). In short, the sequencing reads (Genbank BioProject accession code PRJNA42832) were mapped to the genome of strain EXF-562 using BWA-MEM and sorted and deduplicated (Li \& Durbin 2009). Variant calling was performed using Genome Analysis Toolkit 3.8 (Alkan et al. 2011) according to 'GATK Best Practices' with the 'hard filtering' option. After discarding all of the variants with depth of coverage outside the range of 700-900, the resulting single nucleotide polymorphisms were used to calculate the dissimilarity distance matrix with the $\mathrm{R}$ package Poppr (Kamvar et al. 2015; R Development Core Team 2017). The Neighbour-Net algorithm as implemented in the $R$ package Phangorn (Schliep et al. 2017) was used for construction of the phylogenetic network, with Hortaea thailandica as an outgroup.

All of the alignments have been submitted to TreeBASE, and are accessible at the following address: http:// purl.org/phylo/treebase/phylows/study/TB2:S24174

\section{Halotolerance and thermotolerance tested on solid and in liquid media}

The strains characterised in this study were line-streak cultivated on MEA medium supplemented with $\mathrm{NaCl}(0$, $5,10,15,20,25$, and $30 \% ; \mathrm{w} / \mathrm{v}$ ), and incubated at $25^{\circ} \mathrm{C}$ for up to 9 weeks. The colonies were then measured and the colony structures characterised, as yeast $(\mathrm{Y})$, filamentous (F), or yeast-filamentous (Y-F). The temperature tolerance of the strains was tested on MEA medium incubated at 5, $10,15,25$ and $37^{\circ} \mathrm{C}$ for up to 2 weeks. Growth was recorded as good, weak, or no growth.

For testing of the growth at different $\mathrm{NaCl}$ concentrations, cells of $H$. werneckii were grown in defined YNB medium (ForMedium, Norfolk, UK): 0.17\% (w/v) yeast nitrogen base, $0.5 \%(\mathrm{w} / \mathrm{v})$ ammonium sulphate, $2.0 \%(\mathrm{w} /$ v) glucose, in deionised water, with $\mathrm{pH}$ adjusted to 7.0, and supplemented with $5,10,15,20$, and $25 \% \mathrm{w} / \mathrm{v} \mathrm{NaCl}$, at $24^{\circ} \mathrm{C}$ and $37^{\circ} \mathrm{C}$. The tests were performed in 96-well microtiter plates, in a $200 \mu \mathrm{L}$ volume, with 16 replicates per strain. Each well was inoculated with $10 \mu \mathrm{L}$ cell suspension $\left(10^{7}\right.$ cells $\left./ \mathrm{mL}\right)$ obtained from 5 -day-old cultures grown on Yeast Extract Peptone Dextrose (YPD) agar (1\% yeast extract, $2 \%$ peptone, $2 \% \mathrm{D}$-glucose, $1.6 \%$ agar). The microtiter plates were sealed with parafilm and incubated for up to 2 weeks. Growth was followed daily by measuring the optical density at $590 \mathrm{~nm}$.

\section{Morphological studies}

Strains were streak plated from single colonies onto MEA without $\mathrm{NaCl}$ or supplemented with $10 \% \mathrm{NaCl}$ $(\mathrm{MEA}+10 \% \mathrm{NaCl})$, onto potato dextrose agar (PDA), and onto oatmeal agar (OA), and incubated at $24{ }^{\circ} \mathrm{C}$ for up to 2 weeks. The diameters of the colonies and their colours and structures were recorded after 2 weeks of incubation. The colour codes used to describe the colony colours were from Kornerup and Wanscher (1978). For the microscopy study, slides were prepared from 2-week-old cultures using the media indicated above, from the colony centre and the colony margin, and were mounted in $60 \%$ lactic acid. The preparations were observed under light microscopy (BX-51, Olympus, Tokyo) with interference contrast optics. Micrographs were recorded with a digital camera (DP73, Olympus). Conidia (one celled, two celled) and hyphae were measured using the micro-imaging software cellSens (Olympus). These measurements are given as means \pm standard deviation from $\geq 50$ conidia and $\geq 10$ hyphae.

\section{Use of carbon and nitrogen sources tested with a Biolog system}

For phenotypic assessment of five strains of Hortaea werneckii (EXF-151, EXF-225, EXF-2788, EXF-6651, and EXF-10513) three different sets of phenotypic microarrays were used (Biolog Inc., Hayward, CA): PM1 MicroPlate and PM2A MicroPlate for carbon metabolism, and PM3B MicroPlate for nitrogen metabolism. The cultures were grown on YPD medium for 5 days at $24{ }^{\circ} \mathrm{C}$.

The cells were then resuspended in sterile water to obtain homogenous suspensions the turbidities of which were adjusted to an absorbance of 0.2 at $590 \mathrm{~nm}$, as described previously (Viti et al. 2015). The inoculation suspensions were prepared by adding $0.25 \mathrm{~mL}$ cell suspension in minimal medium (IFY-0, 1.2 $\times$ ) that lacked sources of either carbon (for PM1, PM2A) or nitrogen (for PM3B), and one of the two corresponding phenotypic microarray $(12 \times)$ additives and tetrazolium dye mix (DyeMixE; Biolog Inc., Hayward, CA). The phenotypic microarray additives were prepared as sterile $12 \times$ concentrated solutions: for 
PM1 and PM2A, the NPS additive (nitrogen/ phosphorus/ sulphur; $60 \mathrm{mM}$ L-glutamic acid monosodium, $60 \mathrm{mM}$ potassium phosphate monobasic anhydrous, $\mathrm{pH}$ 6.0, $24 \mathrm{mM}$ sodium sulphate); and for PM3B, the CPS additive (carbon/ phosphorus/ sulphur; $1200 \mathrm{mM}$ D-glucose, $60 \mathrm{mM}$ potassium phosphate monobasic anhydrous, $\mathrm{pH} 6.0,24$ $\mathrm{mM}$ sodium sulphate). The phenotypic microarray plates were wrapped with parafilm and incubated at $24^{\circ} \mathrm{C}$ for > 2 weeks, and the absorbance was measured at $590 \mathrm{~nm}$ $\left(\mathrm{A}_{590}\right)$ on CytationI3 Imaging reader employing Gen5 Microplate Reader and Imager Software, both from BioTek Instruments (Bad Friedrichshall, Germany). The absorbance readings were taken after $0,2,7$, and 17 days incubation, and the measurements were compared to the negative control (i.e. inoculated well without carbon and nitrogen source, for PM1-2 and PM3, respectively) and to the measurement of the individual wells before incubation of the phenotypic microarray. The phenotypic microarray tests were interpreted as positive for $A_{590}>0.2$, weak for $A_{590} 0.1$ to 0.2 , and negative for $A_{590}<0.1$. Additionally, the plates were inspected visually to ensure that the change in absorbance corresponded to the change of colour due to the reduction of the tetrazolium dye.

\section{Enzyme activity tests}

The extracellular enzyme activities were tested on solid 2\% agar media (Paterson \& Bridge 1994, Strauss et al. 2001, Brizzio et al. 2007) without $\mathrm{NaCl}$ and with addition of 10 and $20 \% \mathrm{NaCl}$. Conidial suspensions of 7-day-old cultures grown at $24{ }^{\circ} \mathrm{C}$ on MEA were used for three-point inoculation of the culture medium. The cultures were incubated in the dark at $25^{\circ} \mathrm{C}$ for 2 weeks and 4 weeks. Proteolytic activity was determined on $0.5 \%$ skimmed milk agar medium with $0.1 \%$ yeast extract (Biolife). A clear zone in the otherwise opaque medium indicated a positive reaction (Brizzio et al. 2007). Esterase activity was determined on Tween 80 agar medium that consisted of $1 \%$ Tween 80 (polyoxyethylene-sorbitan-monooleate), $1 \%$ peptone (Merck), and $0.01 \%$ $\mathrm{CaCl}_{2} \times 2 \mathrm{H}_{2} \mathrm{O}$. The $\mathrm{pH}$ indicator bromocresol blue $(12.5$ $\mathrm{mg} / \mathrm{L}$ ) was added to the medium before adjusting the $\mathrm{pH}$ to 5.4. After incubation, the cleavage of the ester bonds was detected as a precipitate and a purple halo around colonies in the otherwise yellowish-green medium (Brizzio et al. 2007). The basic medium for the testing $\beta$-glucosidase was $0.67 \%$ YNB (Difco), with the addition of $1 \%$ aesculin (Sigma). If not stated otherwise, the $\mathrm{pH}$ of all of the media was adjusted to 5.5 before pouring. To determine $\beta$-glucosidase activity, filter sterilised $1 \%$ ammonium ferric citrate solution was added to the medium, to a final concentration of $0.02 \%$. Enzymatic activity was recognised by dark brown coloration of the medium (Strauss et al. 2001). The activity of gelatinases was determined in $5 \mathrm{~mL}$ nutrient gelatine tubes with 12\% gelatine (Sigma-Aldrich, Munich, Germany), $0.3 \%$ beef extract (Becton Dickenson, Franklin Lakes, $\mathrm{NJ}$ ), and $0.5 \%$ peptone (Merck, Darmstadt, Germany). After the incubation, the tubes were placed at $4{ }^{\circ} \mathrm{C}$ for $30 \mathrm{~min}$, to allow liquefaction of the gelatine, which was interpreted as a positive reaction (Hankin \& Anagnostakis 1975).

\section{Statistical analysis}

The Biolog data were tested for similarity using the SIMPER test (Euclidean distance index) and the PAST software, version 3.13. The phenotypic data (temperature, ESTs) were subjected to multivariant ordination principal component analysis using the PAST software, version 3.13 (Hammer et al. 2001).

\section{RESULTS}

\section{Cluster analysis}

All of these strains studied were identified as Hortaea werneckii based on ITS sequences and the closest GenBank hits. The ITS sequences were of good quality, whereby the chromatograms had clear peaks and no background noise, with a single exception (EXF-11537), which had three ambiguous positions in both strands. The variant calling for ITS and large subunit (LSU) regions for the strains with sequenced genomes showed that a single genome contained a single variant of the ITS and D1/D2 rDNA sequence (Additional file 1: Table S1). In the D1/D2 domains of $28 \mathrm{~S}$ rDNA, 10 genotypes (A-J) were recognised with the manual inspection of the sequence alignment, as also seen in the UPGMA analysis (Fig. 1). Within this dataset, eight variable sites were identified, which constituted $1.5 \%$ dissimilarity in the 521-nucleotide-long alignment (Table 2). The ITS rDNA sequence alignment was 457 nucleotides long, and the number of variable nucleotides was 23 (dissimilarity 5\%), defining 17 different genotypes (Table 3). UPGMA analysis of the $28 \mathrm{~S} \mathrm{rDNA}$ alignment fully reflected the observed genotypes (Fig. 1), but in the ITS rDNA analysis (Fig. 2), the genotypes D/7 (EXF-647) and D/8 (EXF-161, EXF-2788, EXF-96, EXF-631, EXF-9, EXF10830, EXF-2783) were intermixed with genotype B/7, despite single nucleotide differences. On the other hand, according to D1/D2, the $H$. werneckii ex-type strain (CBS 107.67; EXF-151) formed a single strain genotype $\mathrm{J}$, but for the ITS sequence it belonged to genotype 1 . The phylogenetic analyses of the same datasets (i.e. ITS, D1/D2 28S rDNA) with maximum parsimony resulted in overall poorly supported clades (data not shown). Several genotypes showed paraphyletic relationships and not monophyletic groups. Although analyses of the household genes $B T B$ and $M C M 7$ resulted in overall good quality of the sequences, at specific nucleotide positions in the chromatograms for the majority of the 


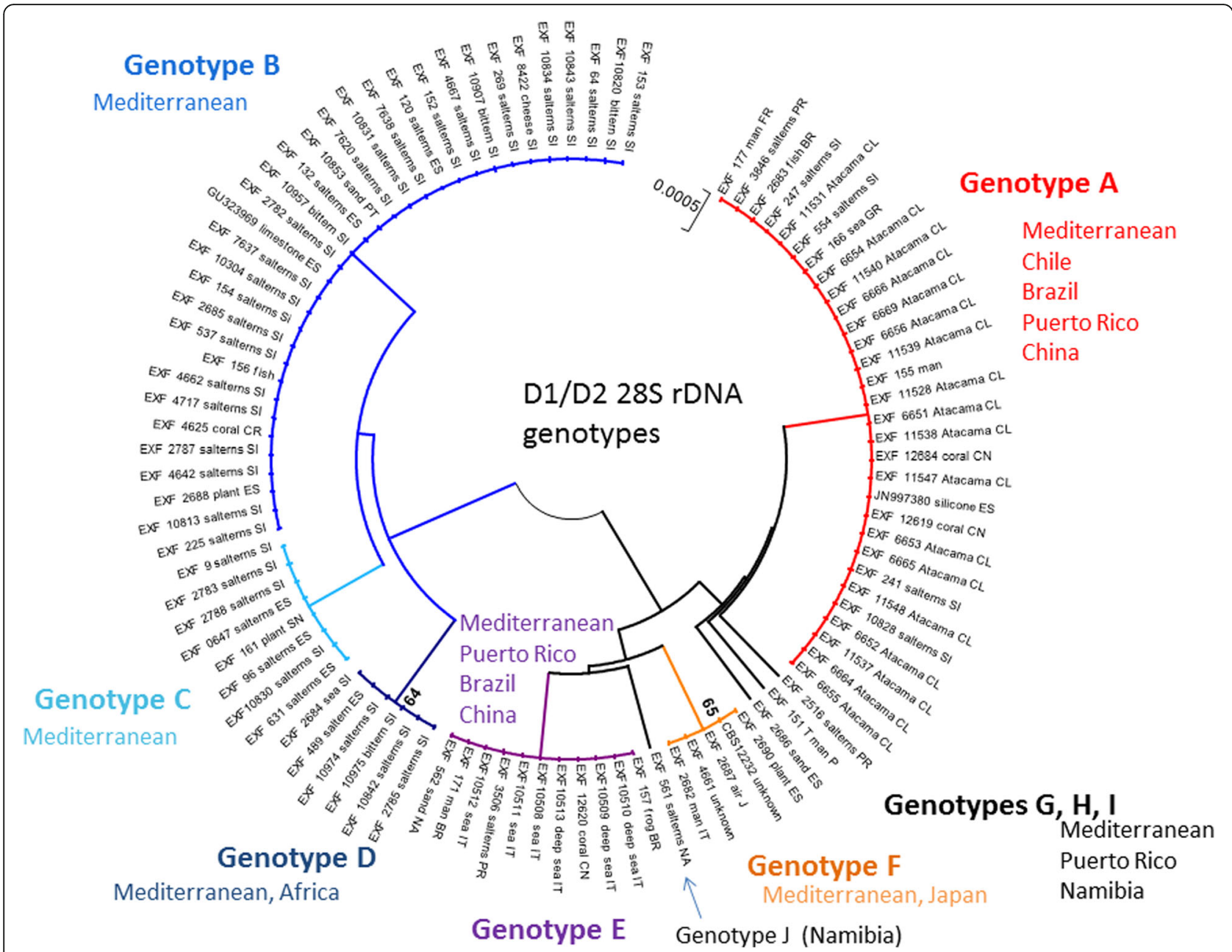

Fig. 1 Unweighted pair group method with arithmetic averages analysis of Hortaea werneckii D1/D2 28S rDNA sequences defines 10 genotypes

Table 2 Variable sites in Hortaea werneckii alignment of partial D1/D2 $28 \mathrm{~S}$ rDNA sequences

\begin{tabular}{|c|c|c|c|c|c|c|c|c|c|c|c|c|}
\hline $\begin{array}{l}28 \mathrm{~S} \\
\text { geno- } \\
\text { type }\end{array}$ & $\begin{array}{l}28 \mathrm{~S} \text { position } \\
\text { / Represen- } \\
\text { tative } \\
\text { EXF strain }\end{array}$ & i & 8 & ले & $\stackrel{\infty}{\infty}$ & $\begin{array}{l}n \\
\infty \\
m\end{array}$ & ปู & 炙 & $\tilde{f}$ & $\hat{d}$ & $\begin{array}{l}\text { corresponding } \\
\text { ITS genotypes }\end{array}$ & $\begin{array}{l}\text { No. of } \\
\text { strains }\end{array}$ \\
\hline A & EXF-554 & C & $\mathrm{T}$ & A & $\mathrm{T}$ & $\mathrm{C}$ & $\mathrm{T}$ & G & C & C & $1,2,3,14,16$ & 27 \\
\hline B & EXF-7638 & C & $\mathrm{T}$ & A & C & C & $\mathrm{T}$ & A & $\mathrm{T}$ & C & $7,8,9,10,12$ & 49 \\
\hline $\mathrm{C}$ & EXF-489 & $\mathrm{T}$ & $\mathrm{T}$ & A & C & C & $\mathrm{T}$ & A & $\mathrm{T}$ & C & 9 & 6 \\
\hline D & EXF-161 & $\mathrm{C}$ & $\mathrm{T}$ & A & C & C & $\mathrm{T}$ & G & $\mathrm{T}$ & C & 7,8 & 9 \\
\hline $\mathrm{E}$ & EXF-562 & C & $\mathrm{T}$ & A & C & C & $\mathrm{T}$ & G & C & C & $4,5,15$ & 11 \\
\hline $\mathrm{F}$ & EXF-2690 & C & C & A & C & C & $\mathrm{T}$ & G & C & C & 4,6 & 5 \\
\hline G & EXF-151 ${ }^{\mathrm{T}}$ & C & $\mathrm{T}$ & G & $\mathrm{T}$ & C & $\mathrm{T}$ & G & C & C & 1 & 1 \\
\hline $\mathrm{H}$ & EXF-2686 & $\mathrm{C}$ & $\mathrm{T}$ & A & $\mathrm{T}$ & C & A & G & C & C & 1 & 1 \\
\hline I & EXF-2516 & C & $\mathrm{T}$ & A & $\mathrm{T}$ & $\mathrm{T}$ & $\mathrm{T}$ & G & C & C & 11 & 1 \\
\hline $\mathrm{J}$ & EXF-561 & C & $\mathrm{T}$ & A & C & $\mathrm{C}$ & $\mathrm{T}$ & G & C & $\mathrm{T}$ & 13 & 1 \\
\hline
\end{tabular}

Legend: Green marked nucleotides represent nucleotide change in a single group or strain strains, double peaks were found in both strands, which led to ambiguity (Fig. 3). On the other hand, unambiguous sequences of both loci were obtained by Sanger sequencing for a number of the strains. The situation was not necessarily the same at both loci, as different strains had, for example, ambiguous sequences in $M C M 7$, but unambiguous sequences in $B T B$.

For the MCM7 gene, these ambiguous sequences were used for computational estimation of the haplotypes, and the estimated haplotypes in most cases matched well to known haplotypes recovered by whole-genome sequencing. Haplotypes from the same strain were positioned in different parts of the intraspecific phylogeny (Fig. 4). The estimated haplotype pairs of strains EXF-2000 (=EXF-225), EXF-4667, EXF-7620, EXF-4662, EXF-152 and EXF-2685 were nearly identical: they had the same phylogenetic position and were collapsed into one leaf per strain in the final tree. Twenty-eight sequences did not contain any ambiguous nucleotide positions. Large 
Table 3 Variable sites in Hortaea werneckii alignment of partial ITS rDNA sequences

\begin{tabular}{|c|c|c|c|c|c|c|c|c|c|c|c|c|c|c|c|c|c|c|c|c|c|c|c|c|}
\hline $\begin{array}{l}\text { ITS } \\
\text { geno- } \\
\text { type }\end{array}$ & $\begin{array}{r}\text { ITS position } \\
1 \\
\text { Represen- } \\
\text { tative } \\
\text { EXF strain }\end{array}$ & o & $\simeq$ & $\stackrel{\infty}{\sim}$ & $\bar{\lambda}$ & $\pi$ & $\bar{\lambda}$ & i & $\bar{m}$ & $\stackrel{\Omega}{\varrho}$ & $\Xi$ & $\stackrel{๑}{\exists}$ & $\Xi$ & $\infty$ & $\overline{\text { I }}$ & $\stackrel{\infty}{\stackrel{\infty}{*}}$ & & 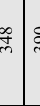 & Zे & न & 昰 & $\tilde{\gamma}$ & $\dot{q}$ & $\begin{array}{l}\text { No. of } \\
\text { strains }\end{array}$ \\
\hline 1 & EXF-151 ${ }^{\mathrm{T}}$ & $\mathrm{T}$ & $\mathrm{T}$ & - & T & C & - & $\mathrm{T}$ & A & $\mathrm{T}$ & $\mathrm{T}$ & A & A & $\mathrm{T}$ & \begin{tabular}{|l|}
$\mathrm{T}$ \\
\end{tabular} & \begin{tabular}{l|l}
$\mathrm{T}$ & \\
&
\end{tabular} & $\mathrm{T}$ & - & $\begin{array}{lll}1 & 0 \\
\end{array}$ & 0 & G & - & \begin{tabular}{|l|}
$\mathrm{T}$ \\
\end{tabular} & 18 \\
\hline 2 & EXF-11531 & $\mathrm{T}$ & $\mathrm{T}$ & - & $\mathrm{T}$ & $\mathrm{C}$ & - & $\mathrm{T}$ & $\mathrm{A}$ & $\mathrm{TT}$ & C & $\mathrm{A}$ & $\mathrm{A}$ & $\mathrm{T}$ & C & $\mathrm{T}$ & & - & c & c & G & - & \begin{tabular}{l|l}
$\mathrm{T}$ \\
\end{tabular} & 6 \\
\hline 3 & EXF-6665 & $\mathrm{T}$ & $\mathrm{T}$ & - & 0 & C & - & $\mathrm{T}$ & A & C & $\mathrm{T}$ & A & A & $\mathrm{T}$ & C & & $\mathrm{T}$ & - & c & c & G & - & \begin{tabular}{l|l}
$\mathrm{T}$ \\
\end{tabular} & 2 \\
\hline 4 & EXF-562 & $\mathrm{C}$ & $\mathrm{T}$ & C & T & $\mathrm{C}$ & G & $\mathrm{T}$ & C & $\mathrm{T}$ & T & A & A & $\mathrm{T}$ & $\mathrm{T}$ & $\mathrm{T}$ & $\mathrm{T}$ & - & $\begin{array}{lll}\Gamma & \mathrm{C}\end{array}$ & c & G & - & $\mathrm{T}$ & 11 \\
\hline 5 & EXF-12619 & C & $\mathrm{T}$ & C & T & C & G & $\mathrm{T}$ & C & C & $\mathrm{T}$ & A & A & $\mathrm{T}$ & $\mathrm{T}$ & $\mathrm{T}$ & T & - & c & c & G & - & $\mathrm{T}$ & 3 \\
\hline 6 & EXF-2682 & $\mathrm{C}$ & $\mathrm{T}$ & C & T & $\mathrm{C}$ & G & $\mathrm{T}$ & C & C & $\mathrm{T}$ & A & G & $\mathrm{T}$ & \begin{tabular}{|l|l|}
$\mathrm{T}$ \\
\end{tabular} & $\begin{array}{ll}\mathrm{T} & \text {, }\end{array}$ & $\mathrm{T}$ & $\mathrm{A}$ & c & c & G & - & \begin{tabular}{|l|l|}
$\mathrm{T}$ \\
\end{tabular} & 3 \\
\hline 7 & EXF-647 & $\mathrm{T}$ & $\mathrm{T}$ & - & T & C & - & $\mathrm{T}$ & A & C & $\mathrm{T}$ & A & $\mathrm{A}$ & $\mathrm{T}$ & $\mathrm{T}$ & $\mathrm{T}$ & $\mathrm{T}$ & - & $\begin{array}{ll}1 \\
\end{array}$ & c & G & C & C & 46 \\
\hline 8 & EXF-161 & $\mathrm{T}$ & $\mathrm{T}$ & - & T & C & - & $\mathrm{T}$ & A & C & $\mathrm{T}$ & A & A & $\mathrm{T}$ & $\mathrm{T}$ & $\mathrm{T}$ & $\mathrm{T}$ & - & c & o & G & $A$ & c & 9 \\
\hline 9 & EXF-489 & $\mathrm{T}$ & $\mathrm{T}$ & - & T & $\mathrm{C}$ & - & $\mathrm{T}$ & $\mathrm{A}$ & C & $\mathrm{T}$ & A & $\mathrm{A}$ & $\mathrm{T}$ & $\mathrm{T}$ & $\mathrm{T}$ & $\mathrm{T}$ & - & \begin{tabular}{l|l}
$\mathrm{T}$ & $\mathrm{T}$ \\
\end{tabular} & c & A & C & C & 7 \\
\hline 10 & EXF-4717 & $\mathrm{T}$ & $\mathrm{T}$ & - & T & C & - & $\mathrm{T}$ & A & C & $\mathrm{T}$ & A & $\mathrm{A}$ & $\mathrm{T}$ & $\mathrm{T}$ & $\begin{array}{ll}\mathrm{T} \\
\end{array}$ & T & - & 10 & c & A & C & C & 1 \\
\hline 11 & EXF-2516 & $\mathrm{T}$ & $\mathrm{T}$ & - & T & C & - & $\mathrm{T}$ & A & C & $\mathrm{T}$ & $\mathrm{A}$ & $\mathrm{A}$ & $\mathrm{T}$ & \begin{tabular}{|l|} 
T \\
\end{tabular} & $\mathrm{T}$ & $\mathrm{T}$ & - & $\begin{array}{ll}\Gamma & 0\end{array}$ & G & G & - & $\mathrm{T}$ & 1 \\
\hline 12 & EXF-2688 & $\mathrm{T}$ & $\mathrm{T}$ & - & T & C & - & $\mathrm{T}$ & A & C & T & A & A & $\mathrm{T}$ & $\mathrm{T}$ & \begin{tabular}{l|l}
$\mathrm{T}$ & ,
\end{tabular} & T & - & $\mathrm{c}$ & c & G & C & C & 1 \\
\hline 13 & EXF-561 & $\mathrm{C}$ & G & C & T & $\overline{\mathrm{A}}$ & G & $\bar{G}$ & C & C & $\mathrm{T}$ & A & A & $\mathrm{T}$ & $\mathrm{T}$ & $\begin{array}{ll}\mathrm{T} & \text {, }\end{array}$ & $\mathrm{T}$ & - & \begin{tabular}{l|l}
$\Gamma$ & 0 \\
\end{tabular} & c & G & - & $\mathrm{T}$ & 1 \\
\hline 14 & EXF-11537 & $\mathrm{T}$ & $\mathrm{T}$ & - & $\mathrm{T}$ & $\mathrm{C}$ & - & $\mathrm{T}$ & A & $\mathrm{T}$ & $\mathrm{Y}$ & $\mathrm{A}$ & A & $\mathrm{T}$ & $\mathrm{Y}$ & \begin{tabular}{l|l}
$\mathrm{T}$ & $\mathrm{Y}$
\end{tabular} & Y & - & $\begin{array}{ll}\Gamma & 0 \\
\end{array}$ & c & G & - & $\mathrm{T}$ & 1 \\
\hline 15 & EXF-12620 & C & $\mathrm{T}$ & C & T & C & G & $\mathrm{T}$ & C & C & $\mathrm{T}$ & G & A & $\mathrm{T}$ & $\mathrm{T}$ & \begin{tabular}{l|l}
$\mathrm{T}$ & \\
&
\end{tabular} & $\mathrm{T}$ & $\mathrm{A}$ & $\begin{array}{ll}\Gamma & 0 \\
\end{array}$ & c & G & - & T & 1 \\
\hline 16 & EXF-3846 & $\mathrm{T}$ & $\mathrm{T}$ & - & T & C & - & $\mathrm{T}$ & A & $\mathrm{T}$ & $\mathrm{T}$ & $\mathrm{A}$ & A & $\mathrm{C}$ & $\mathrm{T}$ & $\mathrm{T}$ & T & - & $\mathrm{c}$ & c & G & - & $\mathrm{T}$ & 1 \\
\hline 17 & EXF-6667 & $\mathrm{T}$ & $\mathrm{T}$ & 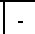 & $\mathrm{T}$ & C & - & $\mathrm{T}$ & $\mathrm{A}$ & $T$ & $\mathrm{~T}$ & A & $\mathrm{A}$ & $\mathrm{T}$ & $\mathrm{T}$ & r & $\mathrm{T}$ & - & \begin{tabular}{l|l}
$T$ & $\mathrm{C}$
\end{tabular} & c & G & - & \begin{tabular}{|l|l|}
$\mathrm{T}$ \\
\end{tabular} & 1 \\
\hline
\end{tabular}

Legend: Green marked nucleotides represent nucleotide change in a single group or strain. Yellow marked nucleotides $(Y)$ represent ambiguous peaks in the sequence, being either Cytosine (C) or Thymine (T)

phylogenetic clusters largely corresponded to clusters of $M C M 7$ genes (Fig. 4, clusters marked 'a' and 'b').

The distances between the strains calculated from single nucleotide polymorphism data and used for the construction of the phylogenetic network (Fig. 5) in general matched the distances estimated using the ITS and D1/D2 rDNA sequences. However, the analysis also showed a substantial reticulation within the phylogenetic history of the strains.

\section{Halotolerance}

Halotolerance was expressed as colony diameters of strains on solid MEA media, without $\mathrm{NaCl}$ and supplemented with 10, 20, 25 and $30 \%(\mathrm{w} / \mathrm{v}) \mathrm{NaCl}$. This was measured after 9 weeks of incubation, and the data are given in Additional file 1: Table S2. All of the strains grew without $\mathrm{NaCl}$ and at up to $20 \% \mathrm{NaCl}$ within these 9 weeks, with the exception of two strains (i.e., EXF-3846, EXF-2687), which did not grow at $20 \% \mathrm{NaCl}$.

The growth optimum was at $10 \% \mathrm{NaCl}$, which was typically seen as filamentous colonies, with yeast-like cells in the central part. EXF-120 grew as yeasts without $\mathrm{NaCl}$ and at $20 \% \mathrm{NaCl}$, but showed filamentous immersed growth with $10 \% \mathrm{NaCl}$. All other strains were either filamentous on all of the media (representative strain EXF-4717), or formed numerous filamentous sectors within initial yeast colonies without $\mathrm{NaCl}$ (representative strains EXF-96, EXF-467), or only filamentous growth without sectors on media with $\mathrm{NaCl}$ (representative strains EXF-467, EXF-96) (Fig. 6).
Over the 9 weeks of incubation, only a few of the strains (EXF-247, EXF-554, EXF-156, EXF-269, EXF-4667, EXF-489, EXF-647, EXF-3506) grew at $30 \% \mathrm{NaCl}$, to produce colonies of 1-2 $\mathrm{mm}$ diam. Some additional strains grew at $30 \% \mathrm{NaCl}$ after longer incubation periods (data not shown). The largest differences identified by SIMPER tests between the genotypes were in the colony diameters on $\mathrm{MEA}+20 \% \mathrm{NaCl}$, where the group of strains belonging to LSU/ITS genotype E/4 developed colonies that were 4-fold larger than for the other genotypes. Other strains from this group grew more slowly and did not attain such colony sizes.

\section{Thermotolerance}

The growth of the tested strains on solid MEA media without $\mathrm{NaCl}$ and supplemented with $10 \% \mathrm{NaCl}$ and at different temperatures $\left(5,15,25,37^{\circ} \mathrm{C}\right)$ varied among the strains (Additional file 1: Table S3). Here, some patterns could be correlated with some genotypes, as shown by principal component analysis (Fig. 7). For example, the sea and deep-sea strains from Italy (genotype $\mathrm{E} / 4$ ) grew at $5{ }^{\circ} \mathrm{C}$ to $37^{\circ} \mathrm{C}$ regardless of the addition of $\mathrm{NaCl}$. Their growth at $5^{\circ} \mathrm{C}$ was slow, while it was rapid at $37^{\circ} \mathrm{C}$, and comparable to their growth at $25^{\circ} \mathrm{C}$. However, a human-associated strain (EXF-171) from the same genotype $(\mathrm{E} / 4)$ did not grow at $5^{\circ} \mathrm{C}$ and at $37^{\circ} \mathrm{C}$, which might have been due to its degeneration during subcultivation, as it was originally isolated in 1935 . Strains from Atacama, Chile, that belonged to genotypes $\mathrm{A} / 1$ to $\mathrm{A} / 3$ grew at $5^{\circ} \mathrm{C}$, but only with the addition of $10 \%$ $\mathrm{NaCl}$, while they did not grow at $37^{\circ} \mathrm{C}$. The strains 


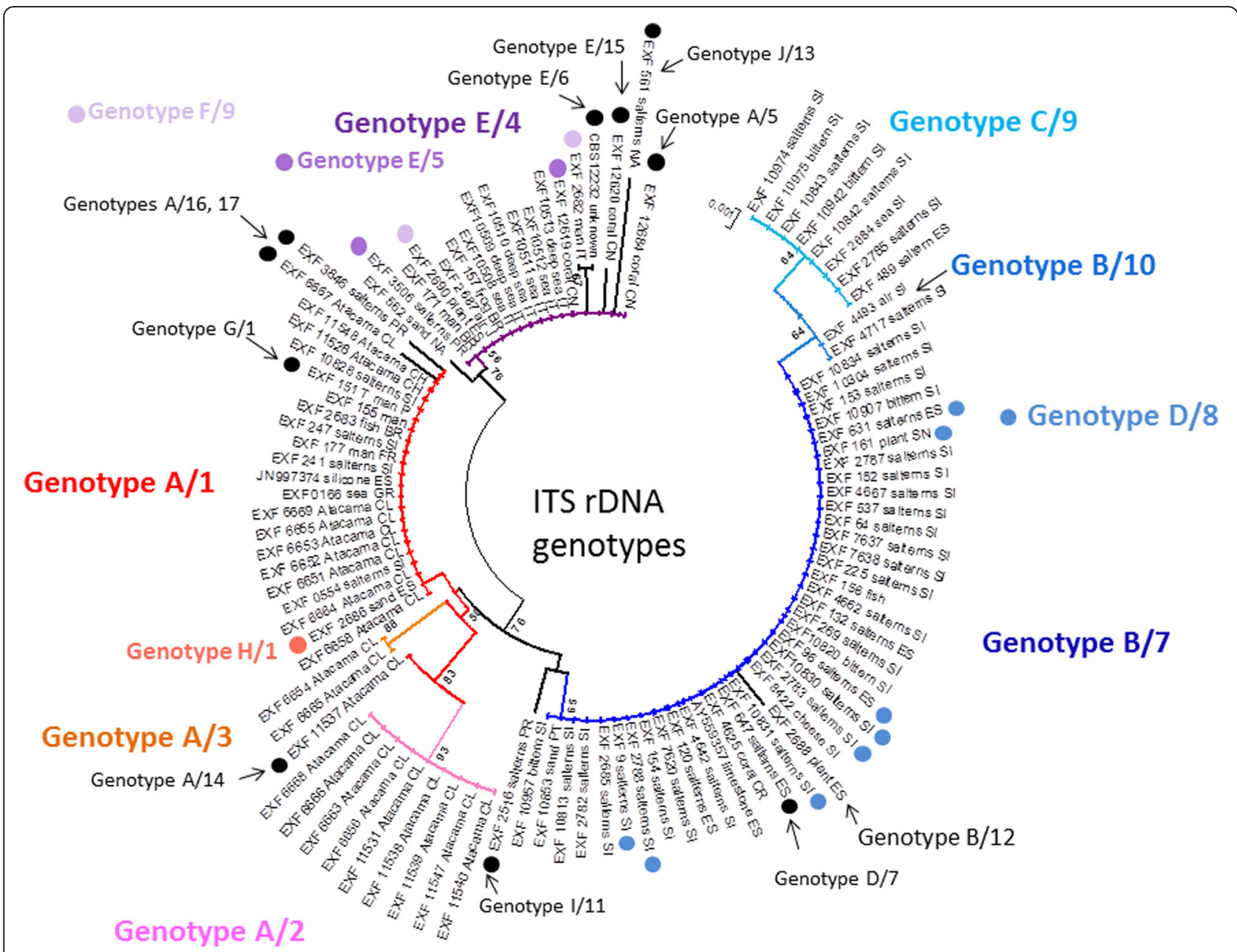

Fig. 2 Unweighted pair group method with arithmetic averages analysis of Hortaea werneckii ITS rDNA genotypes defines 17 genotypes

assigned to genotype $\mathrm{B} / 7$ grew at $15^{\circ} \mathrm{C}$ to $25^{\circ} \mathrm{C}$, without and with $\mathrm{NaCl}$, while they grew at $37^{\circ} \mathrm{C}$ only with the addition of $\mathrm{NaCl}$. The optimum temperature for all tested strains was at $15^{\circ} \mathrm{C}$ to $25^{\circ} \mathrm{C}$.

Strains were also tested for temperature tolerance in liquid media (YNB without and with addition of 5, 10, $15,20,25 \%[\mathrm{w} / \mathrm{v}] \mathrm{NaCl})$. All of these strains grew faster at $25^{\circ} \mathrm{C}$, and grew at $37^{\circ} \mathrm{C}$ with the addition of at least $5 \% \mathrm{NaCl}$ to the medium (Fig. 8), although they reached lower optical densities at this higher temperature $\left(37^{\circ} \mathrm{C}\right.$, $\mathrm{OD}_{590} 0.3 ; 25^{\circ} \mathrm{C}, \mathrm{OD}_{590}$ 3.0). In agreement with the growth tests on solid media, strain EXF-10813 from the deep sea grew well at $37^{\circ} \mathrm{C}$ even without $\mathrm{NaCl}$, although it grew faster with the addition of $\mathrm{NaCl}\left(\mathrm{OD}_{590} 1.5\right)$.

\section{Morphological studies}

The colony morphologies differed substantially among strains and across the culture media. Among 34 strains grown for 2 weeks on MEA, nine different colony morphologies were recorded, which differed in terms of colour, structure and size (Additional file 1: Table S4). The colony colours (according to the Methuen Handbook of Colour) were most often olive-brown (4E7), but also honey-yellow (5D6), mustard-brown (5E6), yellowish grey (2B2), olive yellow (2D7) and blond greyish yellow (4C4). Colony diameters were $2-7 \mathrm{~mm}$, as yeast-like, filamentous, or mixtures of both forms (Figs. 9 and 10, columns A).

In comparison to MEA, the colonies on $\mathrm{MEA}+10 \%$ $\mathrm{NaCl}$ were initially yeast-like, but developed marginal immersed budding mycelium that were visible as filamentous growth, and some became exclusively filamentous (EXF-6656, EXF-3846), while some appeared filamentous regardless of their growth medium (EXF-161, EXF-171), and reached diameters from $0.5-10 \mathrm{~mm}$. The colony colours here were most often olive-brown (4D, E, F), or olive (3E, F4) to golden brown, to yellowish brown (5D, E), dull green (30D3), or even yellowish grey (4B2). 


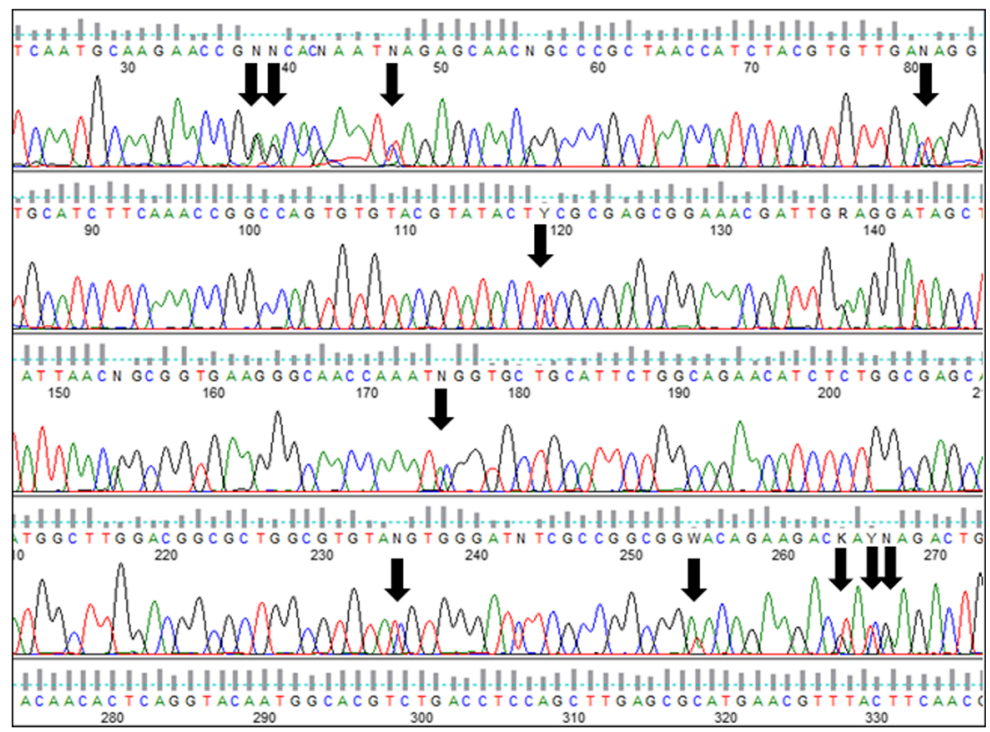

Fig. 3 Chromatogram of the $\beta$-tubulin-coding gene showing the double peaks due to the presence of different gene copies

On OA media, the cultures were dark green (30F5) to black, and reached diameters of $2.5-13.0 \mathrm{~mm}$. The different colonies of the same strain were mostly similar, and were composed of a yeast-like small centre that was surrounded by a wider superficial aerial filamentous margin, although in some cases yeast and yeast/ filamentous colonies developed from a single strain on the same agar plate.

The colonies on PDA were mostly yeast-like and shiny, and reached diameters of $1-9 \mathrm{~mm}$ in 14 days. These were yellowish brown (5F7) to olive-brown (4F4-6), with many darker, or they were coloured similar to those grown on OA (e.g. EXF-161).

The informations on their microscopic structures on these culture media are shown in Fig. 10. In general, the conidial size, shape, and pigmentation depended on the culture medium, and varied among the strains. On all of the culture media, the colonies started to grow as yeasts, and many of them later developed superficial, aerial, or immersed mycelia. The different kinds of hyphae measured from 2.5-15.0 $\mu \mathrm{m}$ diam. The thin hyphae were either composed of long cells (length, 5-15 $\mu \mathrm{m}$ ), or were seen as pseudomycelia that were composed of concatenated twocelled conidia that were constricted at the septa (width, $7 \mu \mathrm{m})$. The thick hyphae were additionally longitudinally septate (Fig. 11). The mycelia had lateral proliferating openings that were either almost sessile or developed short single conidiogenous cells, or even complex conidiophores that developed into synnema-like structures.

On the OA medium, even hyphal clusters that resembled sterile 'pseudo' fruiting bodies were seen (Fig. 12). Conidia developed in annellidic conidiogenesis, and were typically one or two-celled and melanized, with bi-polar budding (Fig. 13). On the OA medium they differed in size and pigmentation. One-celled conidia from the colony centres measured 5.0-8.0 $\times 2.0-$ $4.5 \mu \mathrm{m}$, and from the colony margins 5.5-8.0 $\times 2.0-$ $4.0 \mu \mathrm{m}$. Typical two-celled conidia that aggregated in the colony centres as yeast colonies measured 7.5$15.0 \times 3.0-6.0 \mu \mathrm{m}$, and for some of the strains these were also seen in the marginal parts of the colonies, where they measured 9.0-12.0 $\times 3.0-8.5 \mu \mathrm{m}$ (Additional file 1: Table S5). The mean conidial measurements from the centres of the colonies ( \pm standard deviations) are shown in Fig. 14.

Growth in the form of meristematic clusters was evident for almost all of the strains growing on MEA at $37^{\circ} \mathrm{C}$, and this also appeared in the centres of 14-d-old colonies on PDA and MEA, and for some of the strains also on MEA with 10 to $30 \% \mathrm{NaCl}$ (Fig. 15).

\section{Use of carbon and nitrogen sources}

The 190 carbon assimilation tests were divided into 10 groups according to their main roles in fungal metabolism: pentose catabolic pathway; galactose pathway; starch and sucrose metabolism; uronic acid metabolism; glycolysis and branches; pyruvate metabolism; citrate cycle; amino-acid and protein metabolism; compatible solutes; and aromatic pathways. An additional group of 'other carbon sources' was as defined previously by Nai et al. (2013). Some substrates involved in more than one metabolic pathway were classified into two groups (e.g. sugar alcohols, with D-sorbitol, D-arabitol and L-arabitol classified both as the pentose catabolic pathway and as metabolism of compatible solutes).

Of the five strains tested, good growth was detected in 52 of the carbon assimilation tests (27.4\%) that defined the 


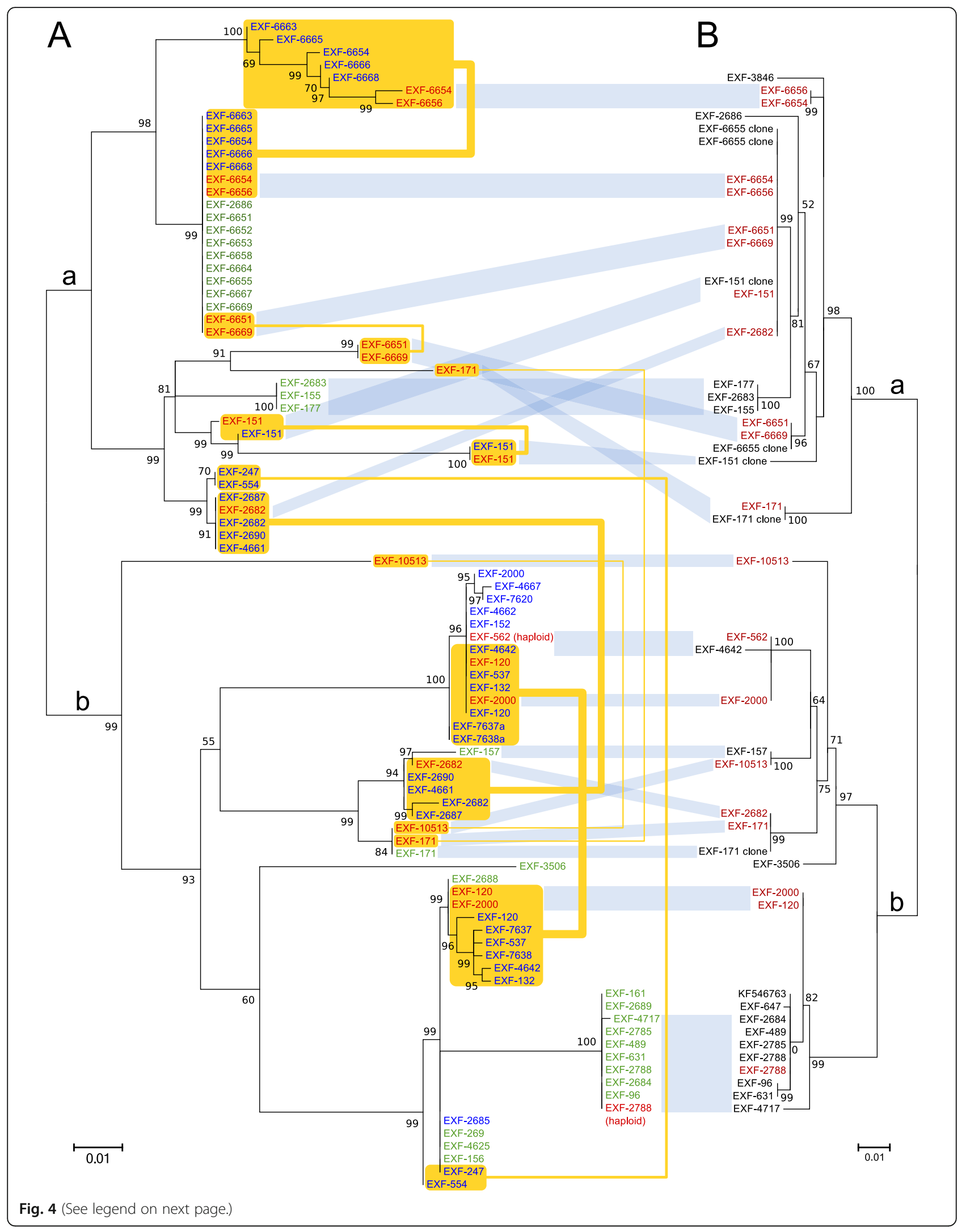


(See figure on previous page.)

Fig. 4 Maximum likelihood phylogenies based on the MCM7 and BTB genes. a The MCM7 gene. The amplicons were amplified by PCR and sequenced by Sanger sequencing. Sequence names without ambiguous nucleotides after sequencing are shown in green. Haplotype estimation was used to separate haplotypes from sequences with ambiguous nucleotides (in blue). Estimated haplotypes with identical phylogenetic positions were collapsed into one sequence. Haplotype sequences recovered from the whole-genome sequences are included for comparison (in red). Haplotype pairs from the same genomes are connected by yellow lines. $\mathbf{b}$ The BTB gene. Only sequences without ambiguous nucleotides and sequences obtained by cloning the amplicons and sequencing individual clones were included in the analysis. Haplotype sequences recovered from the whole-genome sequences are included for comparison (in red). The large phylogenetic clusters observed in both the MCM7 and BTB phylogenies are indicated with ' $a$ ' and 'b'

pentose catabolic pathway, galactose pathway, starch and sucrose metabolism, glycolysis and branches, pyruvate metabolism, citrate cycle, compatible solutes, uronic acid metabolism, and other carbon sources. The most efficiently catabolised substrates were those from the pentose (D-ribose, D-xylose, L-arabinose, L-arabitol) and galactose (D-galactose, D-melibiose, D-raffinose) catabolic pathways, glycolysis and branches (D-glucose, D-mannose, arbutin, gentiobiose, laminarin), and metabolism of compatible solutes (D-mannitol, D-trehalose). None of the strains catabolised 46 of the substrates (24.2\%), a large proportion of which belonged to amino-acid and protein metabolism (34.8\%; 16/46) and other carbon sources (32.6\%; 15/46). The use of the remaining amino-acid and protein metabolism sources and the aromatic pathways varied between the strains, and ranged from good to weak, and negative (see Additional file 1: Table S6 for details).

The catabolism of nitrogen was tested on 95 nitrogen sources that were divided into eight groups: simple nitrogen sources; amino acids and derivates; primary amines; amides; amino sugars; nucleobases and derivates; aminated alkenes; and dipeptides. The strains grew well on the majority of these nitrogen substrates (from
68 to $90 \%$ of the tests), except for EXF-2788, which grew well only on 31 (32.6\%) of these nitrogen substrates, and did not grow on 40 (42.1\%) of them (Additional file 1: Table S7). Also, the SIMPER test revealed that the lack of catabolism of numerous nitrogen sources by this strain (e.g. methylamine, D-glutamic acid, L-histidine, N-butylamine, uric acid, D-alanine, ethylamine, cytosine, cytidine, D,L- $\alpha$-amino-N-butyric acid, L-methionine, uridine) contributed the most to its separation from the remaining strains. All of the strains showed little or no catabolism of amino-acid D-lysine, primary amine ethylenediamin, amino sugars $\mathrm{N}$-acetyl-D-mannosamine and $\mathrm{N}$-acetyl-D-galactosamine, and nucleobases thymidine, thymine and uracil.

\section{Enzymatic tests}

The results of the enzymatic tests are given in Additional file 1: Table S8. The proteolytic activities on casein were positive or weak for around $60 \%$ of the strains, and became weaker or were lost with addition of 5 and $10 \% \mathrm{NaCl}$ at $25^{\circ} \mathrm{C}$; at $37^{\circ} \mathrm{C}$, only $30 \%$ of the strains were positive. The proteolytic activities on gelatine were generally positive and became

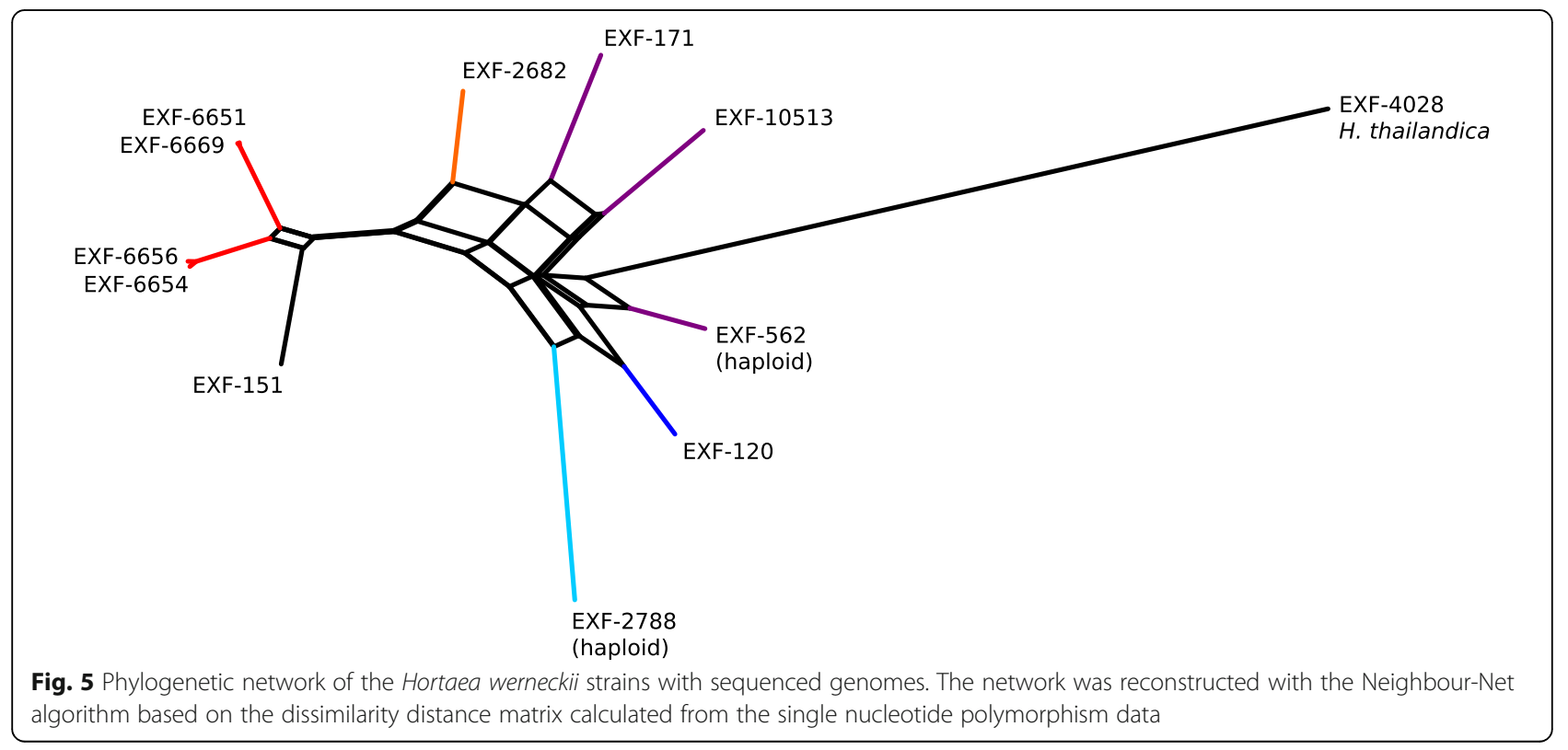




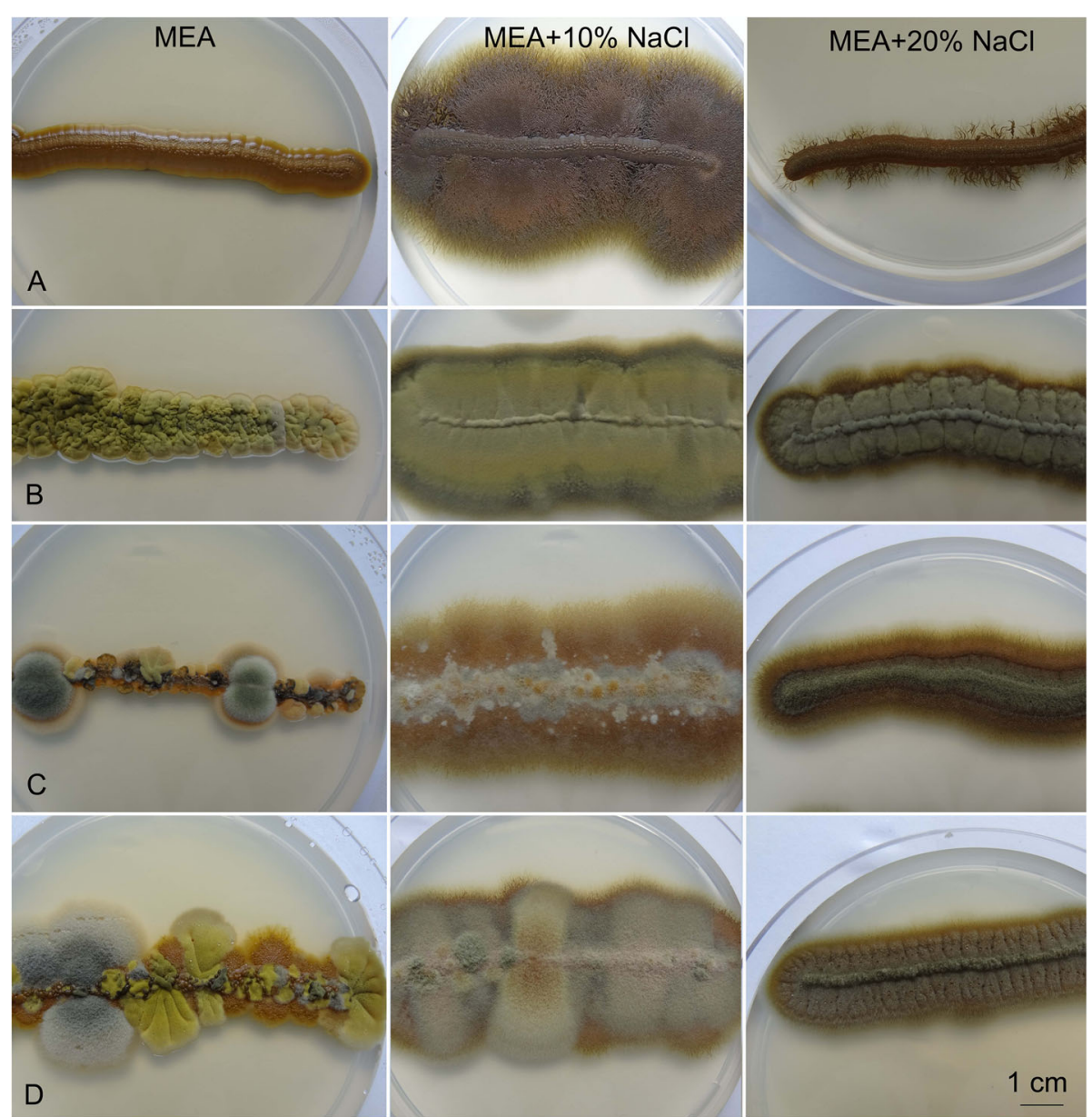

Fig. 6 Morphology of Hortaea werneckii cultures on malt extract agar (MEA) without and with added NaCl. Left: MEA; middle: MEA + 10\% NaCl; right: $\mathrm{MEA}+20 \% \mathrm{NaCl}$. a EXF-120. b EXF-4717. c EXF-467. d EXF-96. Bar $=1 \mathrm{~cm}$ (for all)

stronger with the addition of $\mathrm{NaCl}$. The esterase activities were positive for all of the strains at $25^{\circ} \mathrm{C}$, even in the presence of 5 and $10 \% \mathrm{NaCl}$, while at $37^{\circ} \mathrm{C}$, addition of $10 \% \mathrm{NaCl}$ (but not $5 \% \mathrm{NaCl}$ ) largely inhibited the esterase activity.

\section{DISCUSSION}

Black yeasts are a polyphyletic group of melanized polymorphic fungi that can grow in yeast-like and filamentous forms. They can express an 'extremophilic ecotype' (Gunde-Cimerman et al. 2000) that is characterised by thick melanized cell walls, slow, often meristematic growth, and proliferation with endoconidiation. Globally, black yeasts populate different extreme environments, from hypersaline coastal salterns worldwide (Gunde-Cimerman \& Zalar 2014), to surfaces and subsurfaces of rocks at high or low temperatures (Wollenzien et al. 1995, Selbmann et al. 2005) and Arctic glacial ice (Gunde-Cimerman et al. 2003). Many are involved in opportunistic infections, which demonstrates a certain coherence between extremotolerance and opportunism (de Hoog et al. 2005, Teixeira et al. 2017).

Hortaea werneckii is a cosmopolitan, extremely halotolerant, black yeast without a known sexual morph. Due to its phenotypic plasticity, historically, $H$. werneckii was placed in numerous genera (e.g. Cladosporium, Exophiala, Dematium, Pullularia, Aureobasidium, Sarcinomyces, Pheoannellomyces) before being ascribed to a new genus, due to its special method of conidiogenesis (Nishimura \& Miyaji 1984), of which it was designated the type species. Two additional, so far rarely isolated, species have been described in the last decade: $H$. acidophila from lignite (Hölker et al. 2004) and acidic saline soils (Hujslova et al. 2010); and H. thailandica originally described from plant material in Thailand (Crous et al. 2009), and was later also found in subglacial ice on Svalbard (Zalar \& Gunde-Cimerman, unpubl.). However, $H$. acidophila was later transferred to the genus Neohortaea in Capnodiales based on its phylogeny for nuclear ribosomal and five household genes (Quaedvlieg et al. 2014). 


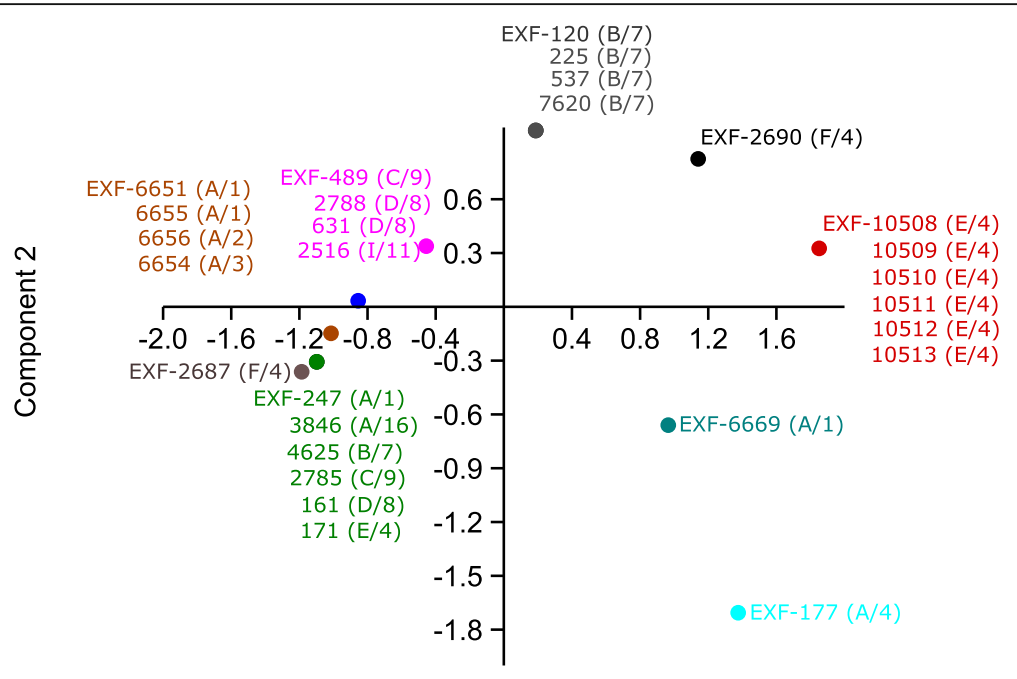

Component 1

Fig. 7 Principal component analysis of Hortaea werneckii growth at different temperatures on solid malt extract agar. Strains with the same temperature patterns are coloured the same

Hortaea werneckii is the causative agent of tinea nigra, and its primary habitat is hypersaline environments and seawater (Gunde-Cimerman \& Zalar 2014). It can also grow in other osmotic environments, as recently demonstrated by its discovery in house dust in Hawaii (Humphries et al. 2017) and on spider webs, walls and sand in arid Atacama caves that have been exposed to marine aerosols (Azúa-Bustos et al. 2010), as indicated above. In the present study, 98 strains were studied that were collected worldwide from natural hypersaline environments and Atacama Desert, and from human, animal and plant sources.

All tested $H$. werneckii strains were halotolerant and grew from 0 to $25 \% \mathrm{NaCl}$, and some strains even grew at $30 \% \mathrm{NaCl}$ (Fig. 6, Additional file 1: Table S2). In agreement with other studies (Gunde-Cimerman et al. 2000, de Hoog et al. 2000, Butinar et al. 2005), these strains grew best from 15 to $25^{\circ} \mathrm{C}$, although some strains grew at $5{ }^{\circ} \mathrm{C}$ and $37^{\circ} \mathrm{C}$, without or with addition of at least 5 to $10 \% \mathrm{NaCl}$ (Fig. 8, Additional file 1: Table S3). This indicated their previously underestimated pathogenic potential (de Hoog et al. 2000).

The morphological data (except for the conidial size) were in agreement with previous descriptions (Nishimura \& Miyaji 1984, de Hoog et al. 2000). In particular, the two-celled conidia were larger than stated in the species description $(7.0-9.5 \times 3.5-4.5 \mu \mathrm{m})$ and varied considerably across the different culture media $(7.5-15.0 \times 2.5-6.5 \mu \mathrm{m}$; Additional file 1: Table S5).

The study of the use of carbon and nitrogen sources for five selected $H$. werneckii strains is the most comprehensive study to date (Additional file 1: Tables S6-S7), and the data are in line with previous data obtained by traditional methods (Zalar et al. 1999, de Hoog et al. 2000). These strains showed various carbon and nitrogen assimilation patterns, with the exception of the haploid strain EXF-2788, which did not catabolise numerous nitrogen substrates.

The extracellular enzymatic activities of four $H$. werneckii strains, which were recently evaluated by Formoso et al. (2015) and Elsayed et al. (2016), were dominated by plant degrading enzymes, including amylase, lipase, esterase, pectinase and/or cellulase, and no animal-related enzymes, like albuminase, keratinase, phospholipase and DNAse. All of the tested strains were urease positive, while caseinase, gelatinase and laccase were variable. The data are in agreement with both of the previous studies (Additional file 1: Table S8), although the enzymatic activities varied considerably between the strains.

$H$. werneckii strains show a unique natural ecology and pathogenic potential, along with high phenotypic and genotypic variability. These aspects were indicated previously and are defined further in this extensive study, and they have raised the question whether a species complex might be concerned (Boekhout et al. 1993, Uijthof et al. 1994, de Hoog et al., 1999, Zalar et al. 1999, Cabañes et al. 2012, Formoso et al. 2015, Elsayed et al. 2016, Marchetta et al. 2018). This also prompted our phylogenetic analysis here. All of the previous studies were based on smaller sets of strains (i.e. from three to 67), and used different genotypic markers and methods, like karyotyping, different PCR fingerprinting techniques, and ITS/28S rDNA sequence variability.

In the present study, the 98 worldwide strains were analysed according to alignments of D1/D2 and ITS 

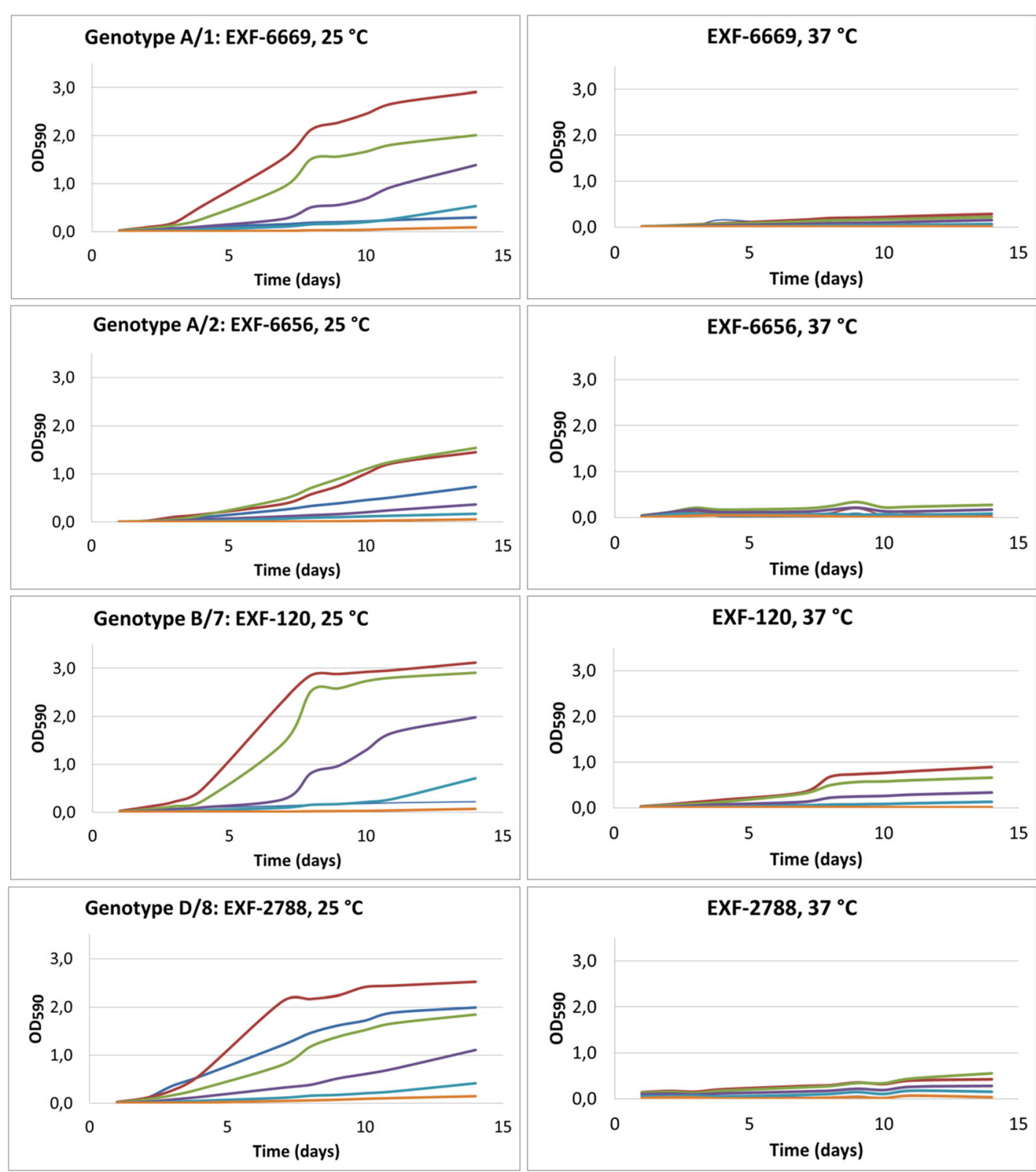

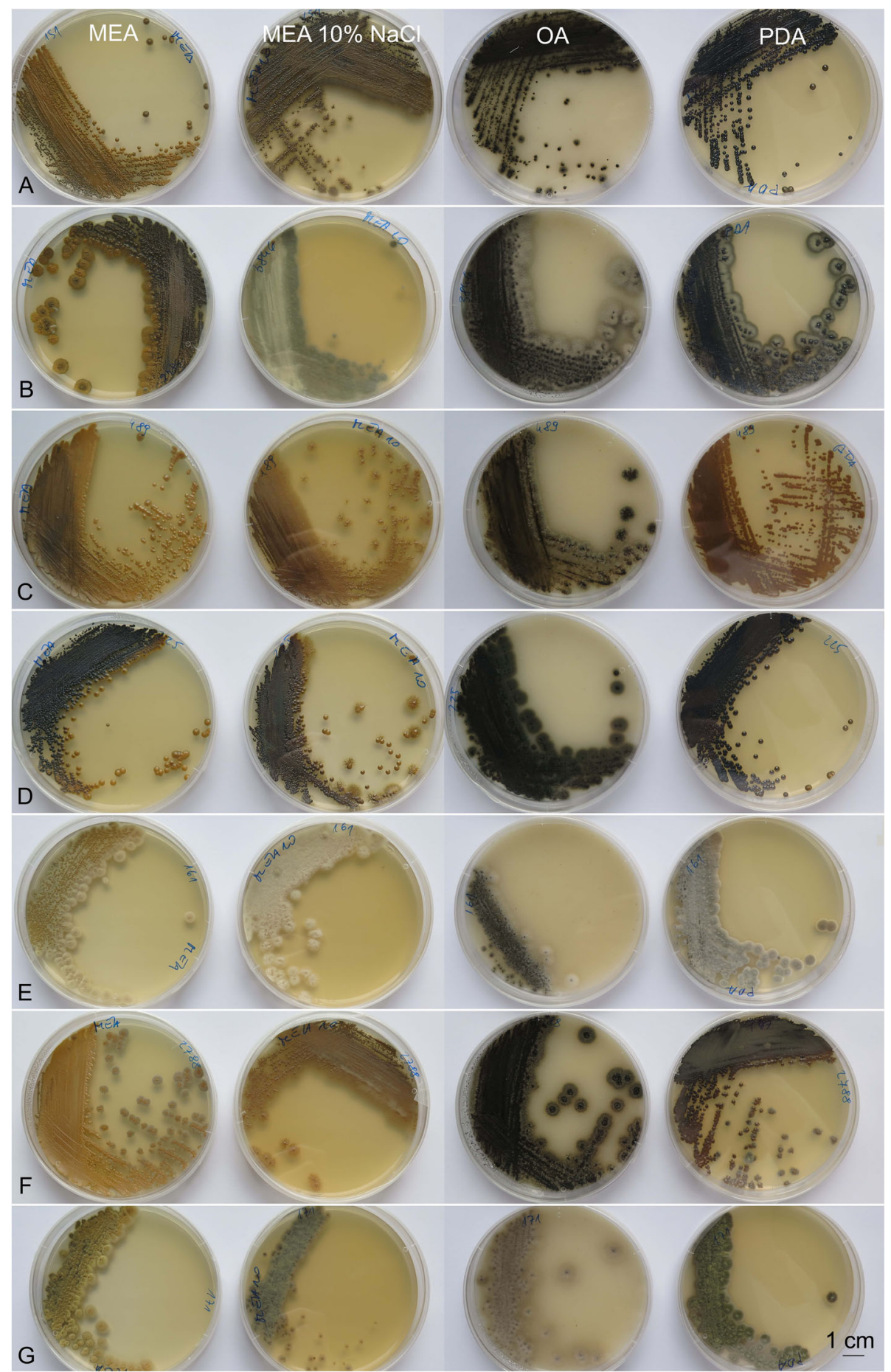

Fig. 9 Macromorphology of Hortaea werneckii strains grown on malt extract agar (MEA; column 1), MEA + 10\% NaCl (column 2), oatmeal agar (column 3) and potato dextrose agar (column 4), for 14 days at $25^{\circ} \mathrm{C}$. a EXF-151 ${ }^{\top}$. b EXF-3846. c EXF-489. d EXF-225. e EXF-161. f EXF-2788. g EXF171. $\mathrm{Bar}=1 \mathrm{~cm}$ (for all)

rDNA sequences. These analyses allowed the definition of 10 and 17 genotypes, respectively, that are based on 1.5 and $5 \%$ dissimilarities, respectively (Figs. 1, 2). In the ITS analysis, several phylogenetically non-informative single point mutations were seen, which resulted in numerous paraphyletic groups in the phylogenetic analysis. The majority of strains that belong to genotype A were derived from tropical, subtropical and southern 


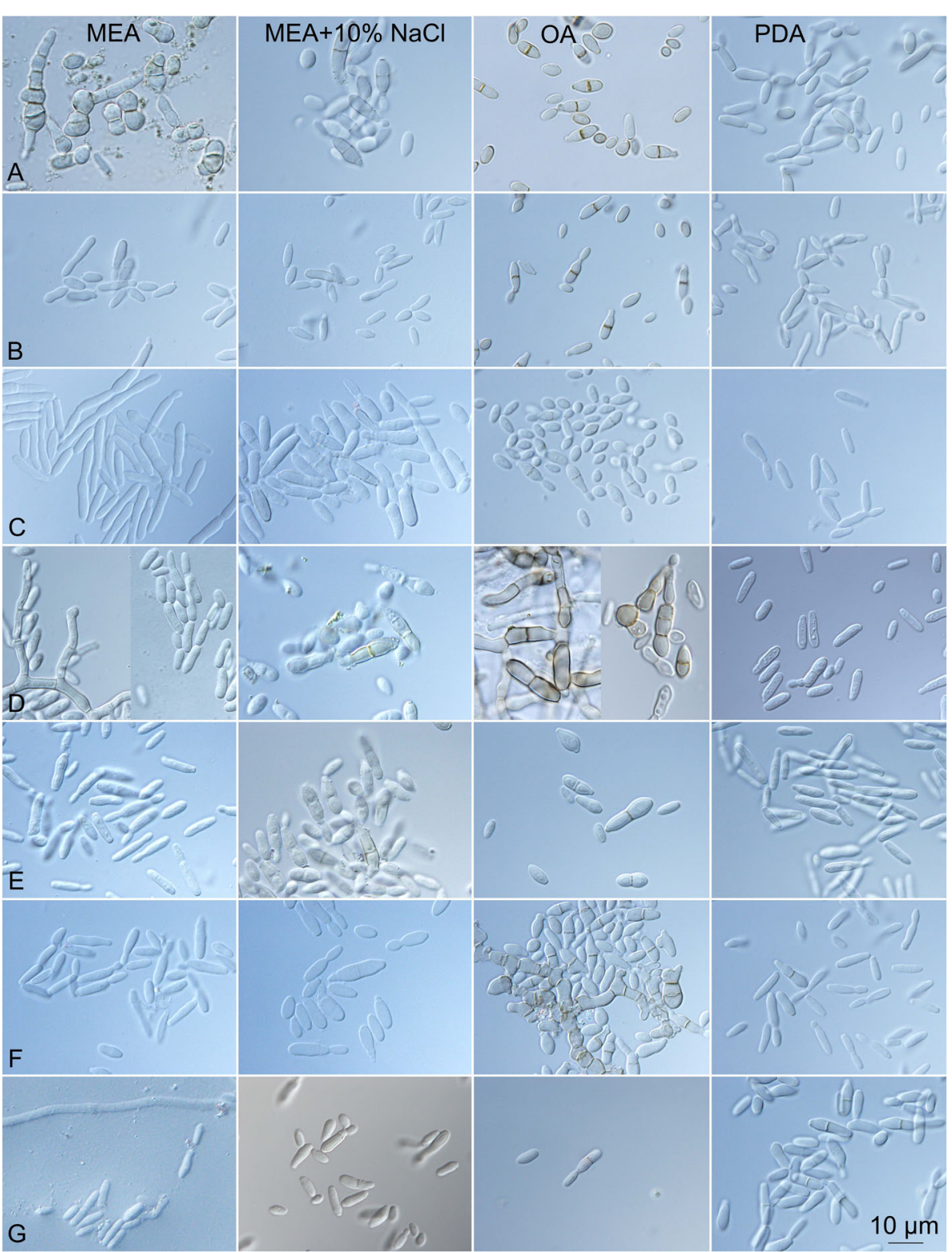

Fig. 10 Micromorphology of Hortaea werneckii strains grown on malt extract agar (MEA) (column 1), MEA + 10\% NaCl (column 2), oatmeal agar (column 3) and potato dextrose agar (column 4) for 7 days at $25^{\circ} \mathrm{C}$. a EXF-151 ${ }^{\top}$. b EXF-3846. c EXF-489. d EXF-225. e EXF-161. f EXF-2788. g EXF171. Bar $=10 \mu \mathrm{m}$ (for all)

hemisphere hypersaline environments, while genotypes $\mathrm{B}$ to $\mathrm{E}$ contained more strains from Mediterranean regions. Although the majority of the strains originated from the Mediterranean area, they were spread (with few exceptions) among almost all of the recognized genotypes. Although genotype $\mathrm{B}$ included some strains from animals (i.e. fish, corals) and plants, animal-related and plantrelated strains were also found in genotype A. The five strains from humans grouped in the 28S/ITS genotypes A/ 1 (France, unknown), E/4 (Brazil), F/9 (Italy) and G/1 (type strain from Portugal), and not in genotype B. Interestingly, all of the strains from the Atacama Desert grouped into genotype A for $28 \mathrm{~S}$ rDNA, but based on ITS, they were divided into four genotypes: $\mathrm{A} / 1, \mathrm{~A} / 2, \mathrm{~A} / 3$, and $\mathrm{A} / 14$. For these strains only, variability was also noted for conidial size: strains of genotype A/1 (EXF-6651, EXF-6655, EXF-6669) had double the conidial width in comparison to strains of genotype A/3 (EXF-6654, EXF-6656) (Figs. 13o-s, 14). Additionally, random representatives of all of the genotypes were highly variable. This intraspecific variability indicates a potentially problematic division into genotypes, as exemplified by the strains associated with tinea nigra. 


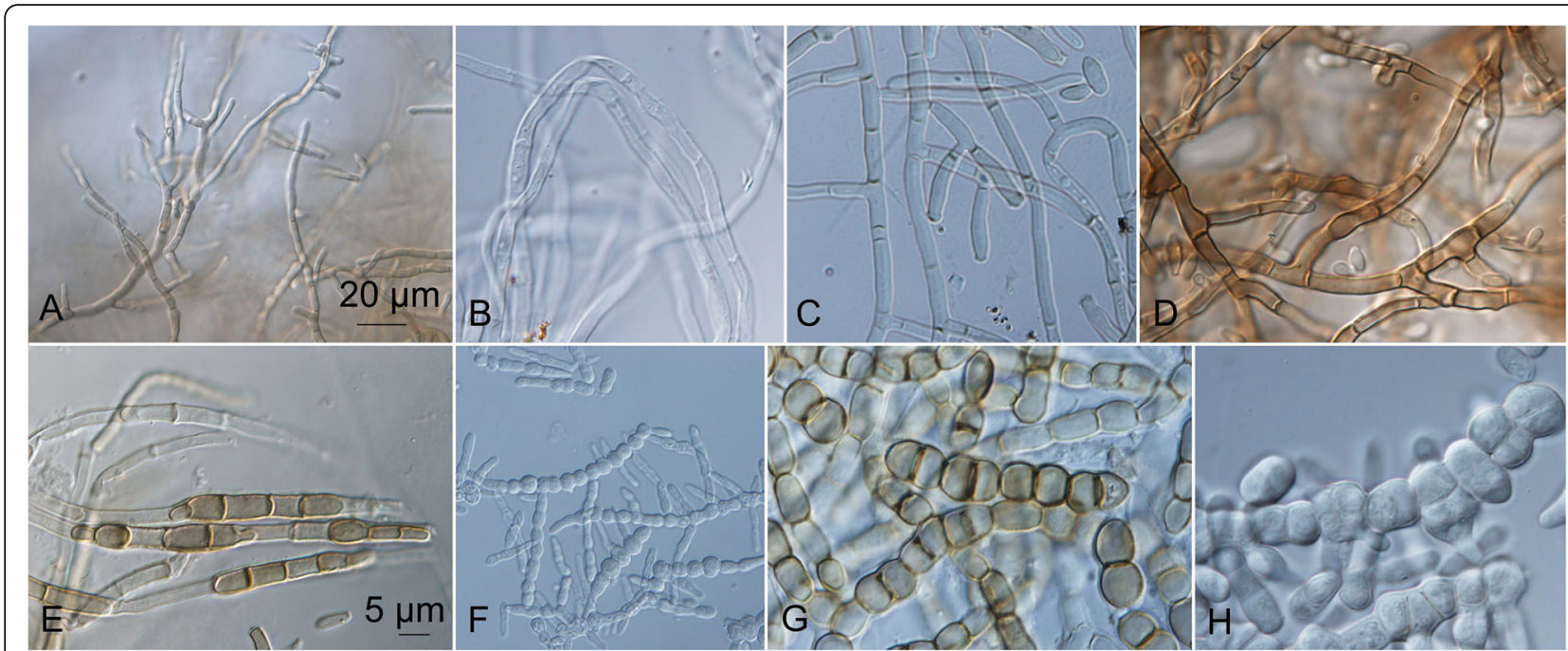

Fig. 11 Different kinds of non-sporulating mycelium produced by Hortaea werneckii cultures after 14 days at $25^{\circ} \mathrm{C}$. a EXF-561: PDA, immersed margin. b EXF-161: oatmeal agar (OA), aerial margin. c EXF-171: potato dextrose agar (PDA), aerial. d EXF-4493: malt extract agar (MEA), immersed centre. e EXF-11537: OA, immersed margin. f EXF-2682: PDA, centre. g EXF-2690: OA, margin. h. EXF-2682: MEA + 10\% NaCl, centre. Bar $=20 \mu m$ $(\mathbf{a}, \mathbf{f}), 5 \mu \mathrm{m}(\mathbf{b}-\mathbf{e}, \mathbf{g}-\mathbf{h})$

The taxonomy of a potential $H$. werneckii species complex was further resolved by Sanger sequencing of the standard taxonomic markers; i.e. the partial $B T B$ and $M C M 7$ genes. Most of the resulting sequences contained ambiguities (Fig. 3). This problem was initially explained by the finding of genome duplication in the single strain where the genome was sequenced (Lenassi et al. 2013, Sinha et al. 2017). A more recent study by Gostinčar et al. (2018) analysed an additional $11 \mathrm{H}$. werneckii genomes, and showed that the majority of the strains were diploid and highly heterozygous. The large divergences within the estimated MCM7 haplotype pairs of individual $H$. werneckii strains matched well the proposed formation of hybrids between heterozygous strains of $H$. werneckii - even though comparative genomics indicated that $H$. werneckii is clonal (Gostinčar et al. 2018). In the analysis here of the $M C M 7$ sequences that included the whole-genome sequence-derived and computationally estimated haplotypes, in three cases the pairs of haplotypes of four or more strains shared the same phylogenetic history (Fig. 4), which might have resulted from the same hybridization event. In addition to this, several such events that involved smaller numbers of strains were indicated. In several cases the phylogenetic distances between the haplotypes were substantial.

The situation shown by the phylograms of the $B T B$ and MCM7 genes depicted in Fig. 4 illustrates the difficulties encountered when investigating intraspecific phylogeny of $H$. werneckii using standard approaches of sequencing molecular markers. In some cases, both copies of the marker genes were amplified. If they did not contain insertions/ deletions, Sanger sequencing produced useful results, although with ambiguous nucleotides in positions that differed between the copies (Fig. 3). After careful investigation of the sequencing chromatograms, the resulting sequence was used for estimation of both haplotypes, a process that was sometimes relatively accurate (e.g. EXF-120), and sometimes unsuccessful (e.g. with the production of two nearly identical haplotypes; such as for EXF-2000/ EXF-225), as seen from the comparisons with the true haplotypes derived from the corresponding whole-genome sequences. In some cases, the sequencing produced good quality sequences with no ambiguities. Theoretically, this can occur if the strain is indeed haploid (e.g. EXF-2788, and possibly other strains in the same cluster), if the two copies of the gene in a diploid genome are identical, or if PCR favours amplification of one copy over the other (e.g. EXF-6669). This last case can lead to an incomplete result - the apparently clear positioning of the species in the intraspecific phylogeny, although the other gene copy might be positioned in a very different part of the gene tree. If both copies are amplified, it is possible to sequence each copy separately by cloning the amplicon and sequencing several clones, as was achieved for $\beta$-tubulin (Fig. 4; strain EXF-6655). However, if the amplification is strongly biased towards one of the copies, this approach is not feasible, as all of the sequenced clones will contain the same copy of the gene.

Interestingly, sequencing the ITS and D1/D2 rDNA markers was not hampered by the heterozygosity that resulted in mixed sequences with ambiguous nucleotide 


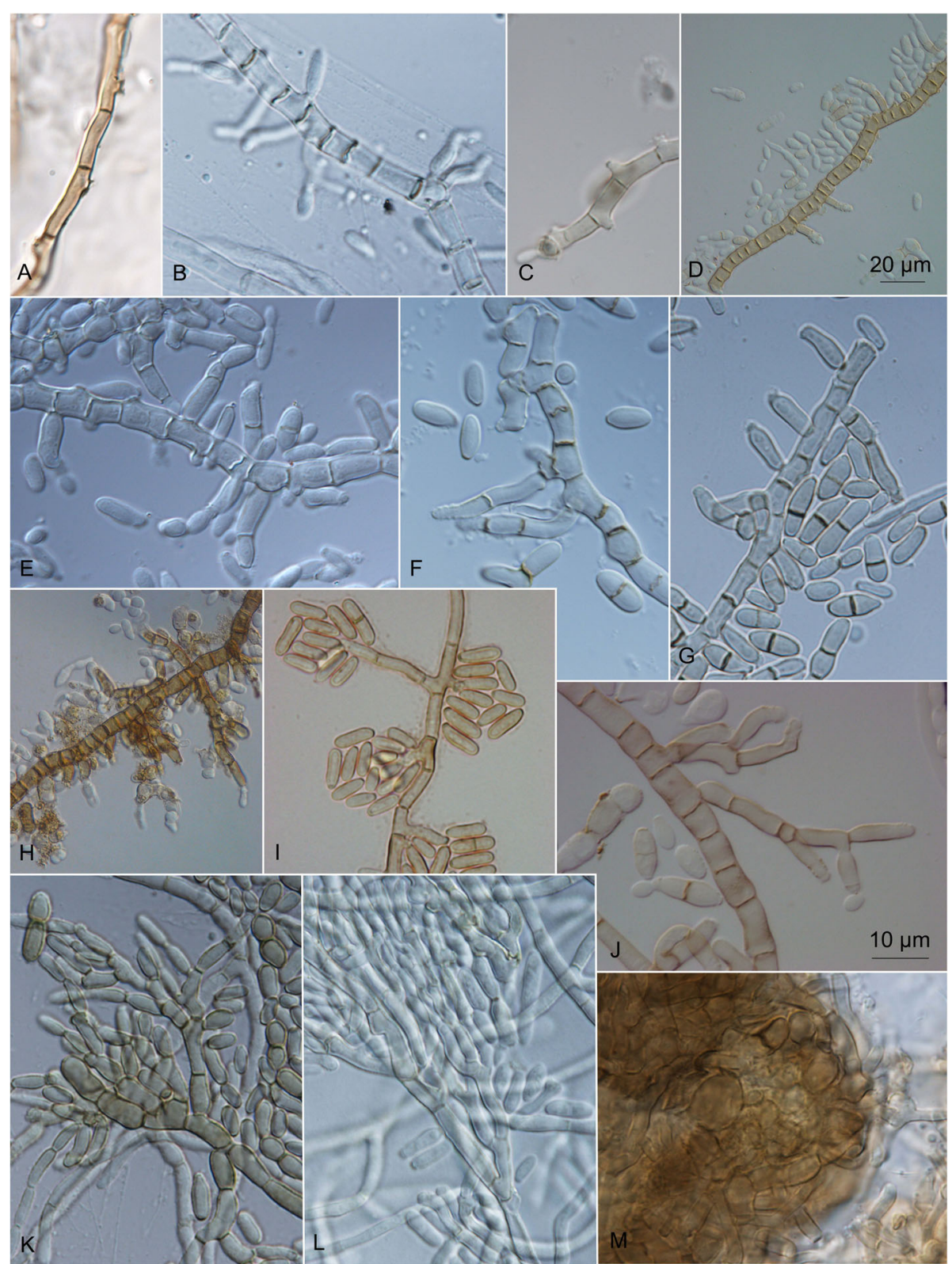

Fig. 12 Sporulating mycelium of Hortaea werneckii strains in cultures after 14 days at $25^{\circ} \mathrm{C}$. a EXF-4493: malt extract agar (MEA), centre. b EXF2682: oatmeal agar (OA), margin. c EXF-489: OA. d EXF-647: MEA + 20\% NaCl. e EXF-151: MEA + 10\% NaCl. f EXF-2687: OA, centre. g EXF-171: potato dextrose agar. $\mathbf{h}$ EXF-6655: MEA + 10\% NaCl, margin. i EXF-6654: OA. j EXF-247: MEA + 10\% NaCl. k EXF-562: OA, margin. I EXF-225: MEA. m EXF-2788: OA. Bar $=20 \mu \mathrm{m}(\mathbf{d}, \mathbf{i}), 10 \mu \mathrm{m}(\mathbf{a}-\mathbf{c}, \mathbf{e}-\mathbf{h}, \mathbf{j}-\mathbf{m})$

positions (Additional file 1: Table S1), as for all of the other genes of $H$. werneckii analysed here. This can be explained by concerted evolution, a process that is responsible for sequence homogenisation of different gene copies in the same genome (Liao 1999). If this is the case, concerted evolution in $H$. werneckii appears to be happening more efficiently in ribosomal sequences than in other genes. A reason for this might be the organisation of the ribosomal DNA in tandem repeats, and the resulting increased opportunity for unequal crossing- over, which has been linked to concerted evolution of the ITS region in fungi (Naidoo et al. 2013). ITS and D1/D2 rDNA phylogenetic markers can thus be used to discern the intraspecific phylogeny of $H$. werneckii without the technical problems that prevent the use of other markers, as discussed above. However, it has to be noted that the phylogeny estimated based on ITS and D1/D2 rDNA markers is a simplified approximation of the real evolutionary history of the species, as it ignores the substantial reticulation of the $H$. werneckii phylogeny (as 


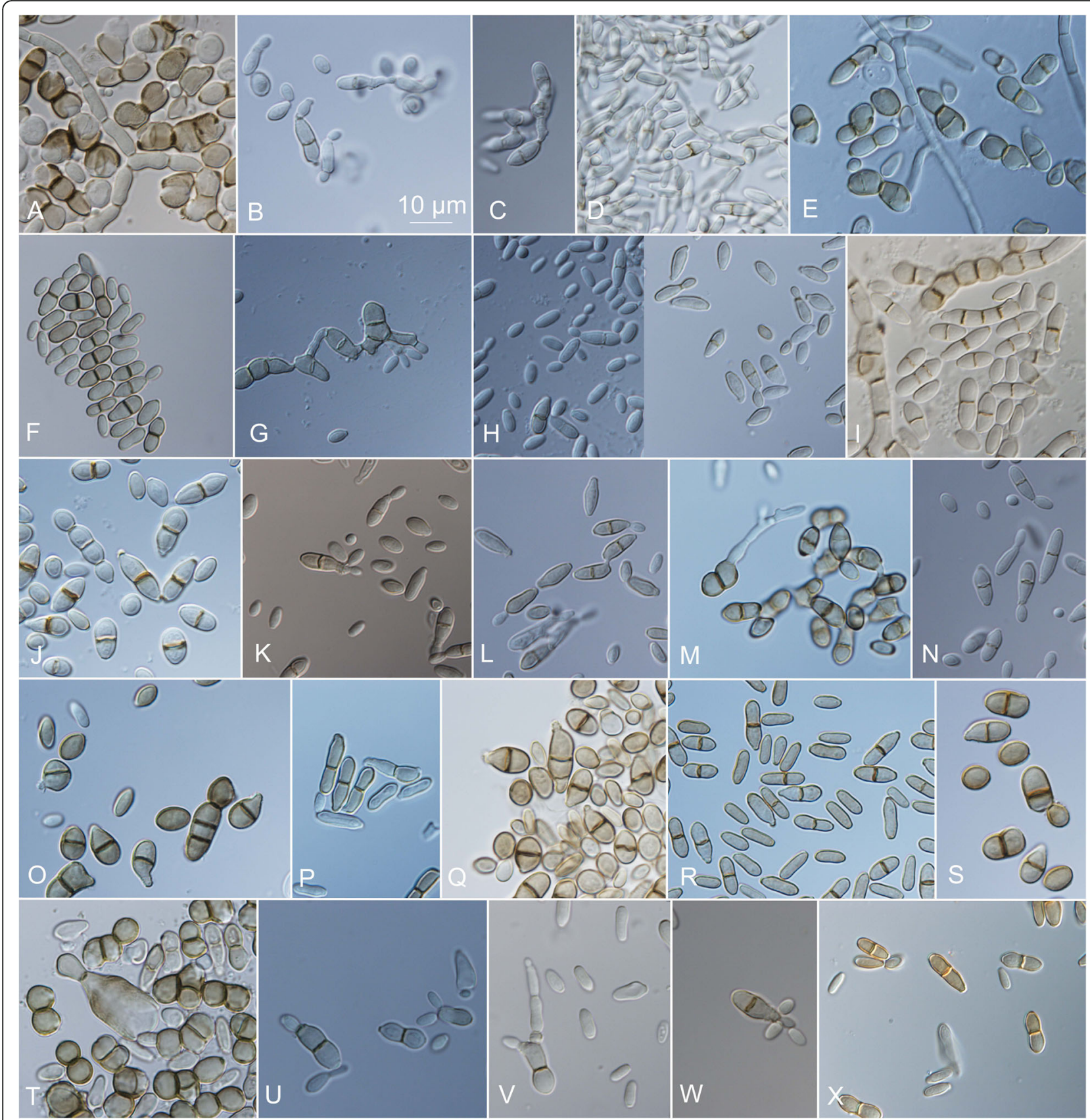

Fig. 13 Conidia of Hortaea werneckii strains grown on oatmeal agar (OA) for 14 days at $25^{\circ} \mathrm{C}$. a EXF-120. b EXF-157. c EXF-161. d EXF-171. e EXF247. $\mathbf{f}$ EXF-562. g EXF-631. h EXF-2516. i EXF-2687. j EXF-2690. $\mathbf{k}$ EXF-2785. I EXF-2788. $\mathbf{m}$ EXF-3506. $\mathbf{n}$ EXF-4625. o EXF-6651. p EXF-6654. $\mathbf{r}$ EXF6656. $\mathbf{s}$ EXF-6669. t EXF-7620. u EXF-10508. v EXF-10512. w EXF-10513. $\mathbf{x}$ EXF-11537. Bar $=10 \mu \mathrm{m}$ (for all)

demonstrated in Fig. 5), which is a consequence of hybridization into persistent and highly heterozygous diploids (Gostinčar et al. 2018).

Hybridization events and changes in ploidy in fungi have been described in other species, but with distinct differences compared to these observations for $H$. werneckii. In Saccharomyces cerevisiae a whole genome duplication event occurred through an ancient hybridization, which was then followed by loss of most of the duplicated regions (Marcet-Houben \& Gabaldón 2015). Apart from this, variations in ploidy and aneuploidy are relatively frequent and are thought to be important for rapid adaptation to environmental changes (Sunshine et al. 2015). Interspecies hybrids have been described in fungi as well, and while most of these are in species only known as asexual morphs, Zygosaccharomyces parabailii was shown to have regained 


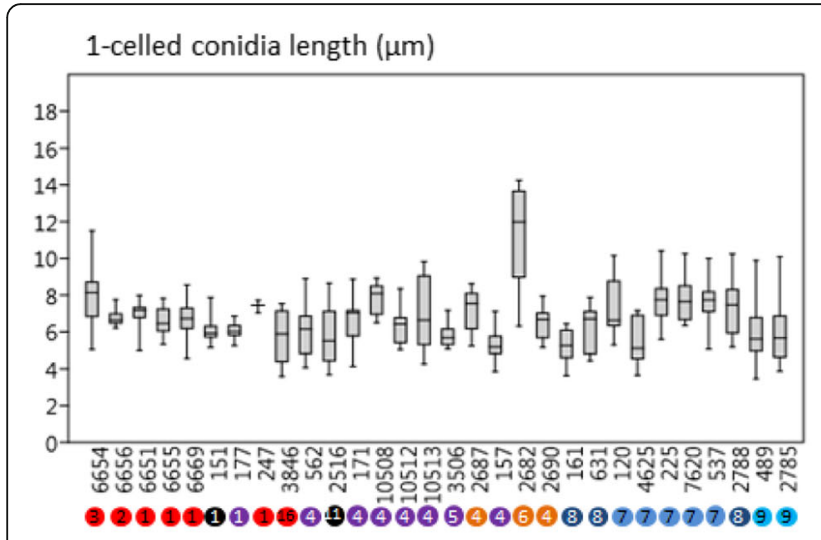

2-celled conidia length $(\mu \mathrm{m})$

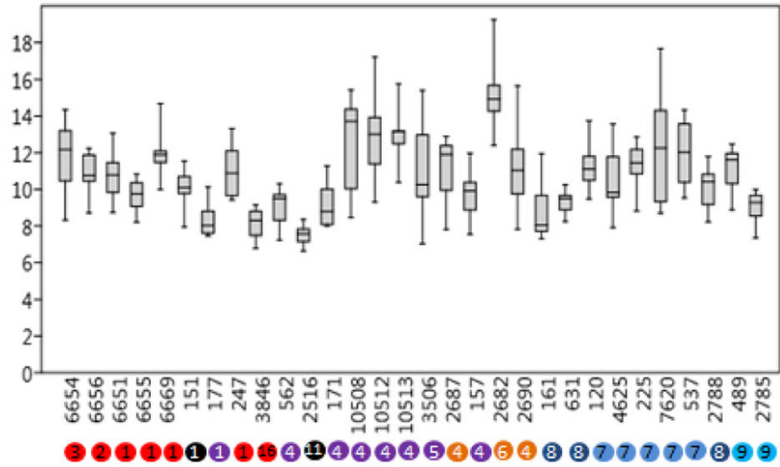

2-celled conidia width $(\mu \mathrm{m})$

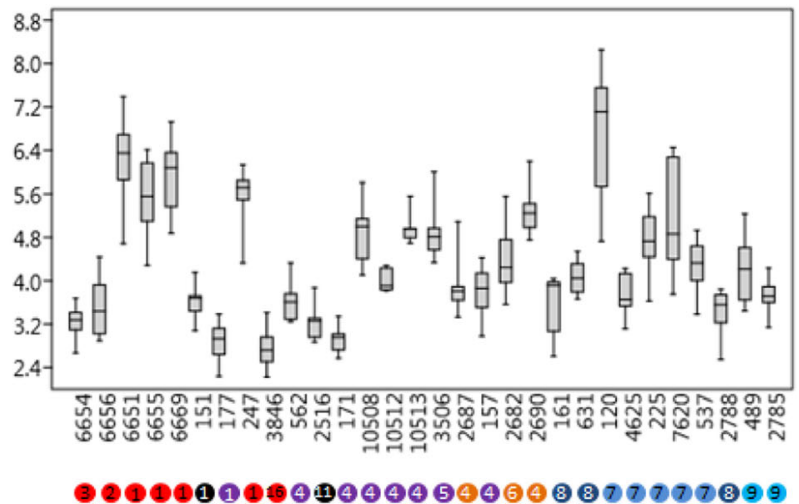

Fig. 14 Measurements of the 1-celled and 2-celled conidia of Hortaea werneckii strains. Data are means \pm standard deviation. 285 rDNA genotypes are indicated as letters (genotype A, red; genotype B, C, D, blue; genotype E, violet; genotype F, orange; miscellaneous LSU genotypes, black), and ITS genotypes as numbers 1-16

fertility by inactivation of one mating-type locus (Ortiz-Merino et al. 2017). Another well-studied example is Candida albicans, a predominantly diploid species that nevertheless has a high degree of genome plasticity, where frequent losses of heterozygosity and gross chromosomal rearrangements might result in aneuploidy (McManus \& Coleman 2014). Although reproduction in C. albicans is predominantly clonal, this species can also use a parasexual cycle that involves the formation of tetraploid progeny from the mating of diploid parents, the former of which subsequently revert to diploidy by concerted chromosome loss (Bennett \& Johnson 2003). While double peaks on sequencing chromatograms can be easily dismissed as a result of suboptimal sequencing quality, they have been reported in at least one other case - in the yeast genus Ogataea. Here, such double peaks were reported to be an indication of heterozygous diploidy or of the existence of gene paralogues that were both/all amplified with the primer sequences used (Čadež et al. 2013).

The failure to recognise hybrids is not only a danger in terms of serious taxonomic misunderstandings, but can also have implications beyond taxonomy. Inderbitzin et al. (2011a) indicated such implications in the management of pathogenic fungi. In this field, the existence of hybrids was first inferred from credible morphological and genetic evidence, and later based on molecular phylogenetics (Inderbitzin et al. 2011a). Hybrids are known in basidiomycete rust genera (e.g. Tilletia, Melampsora, Cronartium, Puccinia), ascomycetous plant pathogens (e.g. Botrytis, Fusarium, Ophiostoma, Verticillium), yeasts (e.g. Saccharomyces, Candida, Cryptococcus), and endophytic fungi. Hybridizations or recombination of introduced fungal pathogens with related resident fungi often results in strains that can infect an expanded range of hosts (Brasier, 2000). By whole genome sequencing of two strains, Fogelqvist et al. (2018) confirmed the findings of Inderbitzin et al. (2011b) for Verticillium longisporum. Their comparatively large draft genomes compared to other ascomycete fungi studied were composed of two parts, where one lineage was more ancient than the part that was more closely related to $V$. dahliae.

The pattern of intraspecific hybridization seen here in $H$. werneckii, which is otherwise a clonal species, represents an unusual strategy that to the best of our knowledge has not been described in other fungi to date. Enriching the genomic toolbox with two different copies of the same 


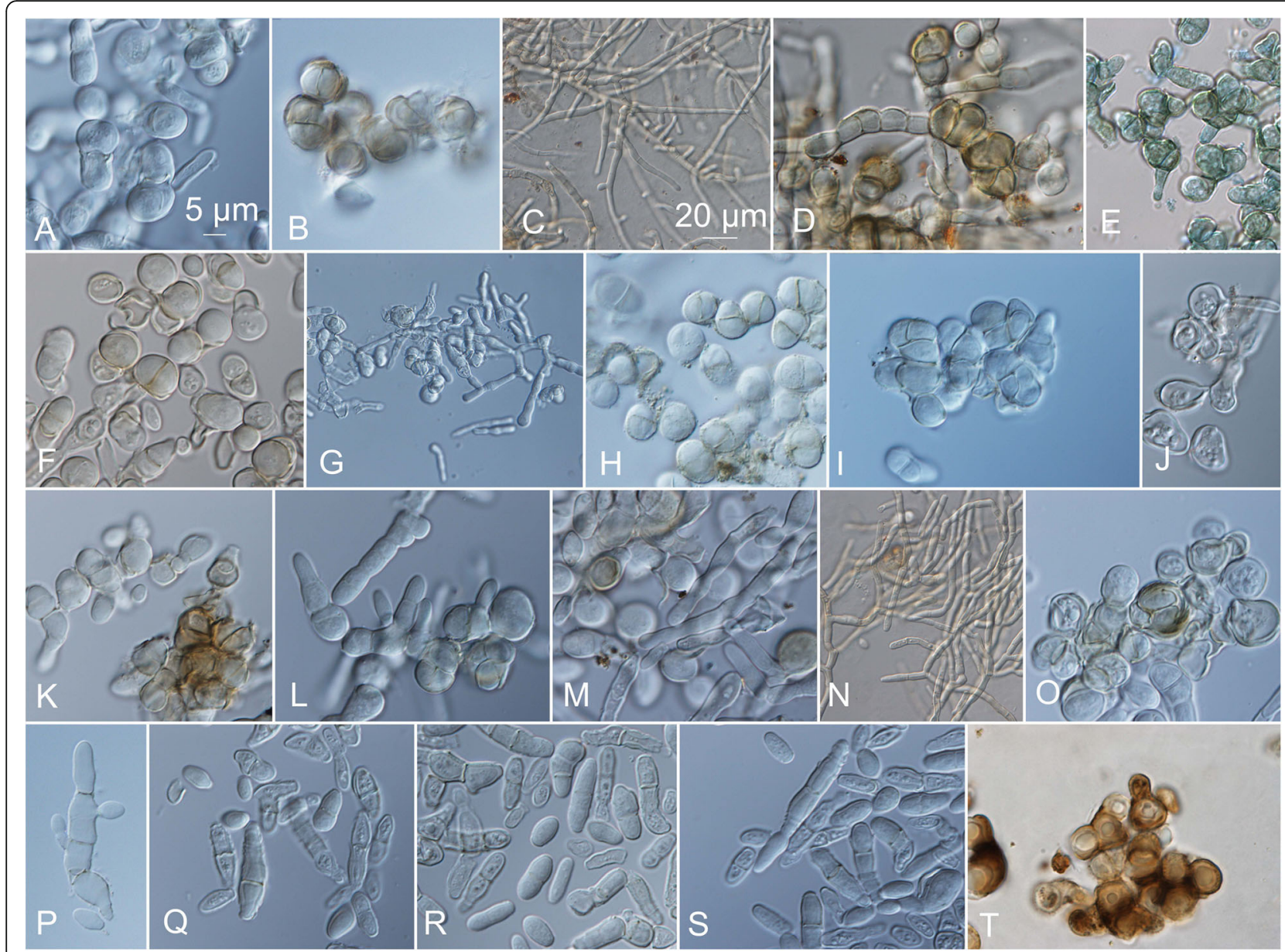

Fig. 15 Growth of Hortaea werneckii strains after 21 days at $37^{\circ} \mathrm{C}$ on solid malt extract agar (MEA) medium supplemented with $10 \% \mathrm{NaCl}$ (if not indicated otherwise). a, b EXF-120. c, d EXF-151 ${ }^{\top}$. e EXF-151 in liquid YNB. f EXF-157. g EXF-225. h EXF-269. i EXF-537. j EXF-561. k EXF-631. I EXF-2690. $\mathbf{m}$ EXF-3506. $\mathbf{n}$ EXF-6651. o, p EXF-7620. q EXF-10508 (on MEA). $\mathbf{r}$ EXF-105012 (on MEA). $\mathbf{s}$ EXF-10513. t EXF-11537 (on MEA). Bar $=5 \mu \mathrm{m}(\mathbf{a}, \mathbf{b}, \mathbf{d}-\mathbf{f}, \mathbf{h}-\mathbf{m}, \mathbf{o}-\mathbf{t}), 20 \mu \mathrm{m}(\mathbf{c}, \mathbf{g}, \mathbf{n})$

gene, or combining traits that are otherwise combined via sexual reproduction, might promote survival under extreme environmental conditions. However, the possible benefits of heterozygous diploid genomes for the survival of $H$. werneckii remain to be investigated. The presence of four ITS genotypes in a small area was seen for the Atacama cave strains, where some diploid strains were almost identical in one haploid subgenome, but differed substantially in the other haploid subgenome (Gostinčar et al. 2018). This makes $H$. werneckii an intriguing extremotolerant experimental system with persistent stable haploid and diploid strains and little, if any, noise introduced by sexual recombination.

\section{CONCLUSIONS}

Ongoing recent trends in fungal taxonomy that are supported by powerful phylogenetic multi-locus analyses encourage descriptions of new species, which can often be based on only a single strain, or a few strains, and/or a few nucleotide differences. When using a limited set of strains, the decisions to recognise cryptic species have to be taken with great caution, as these analyses can be seriously misleading. The present study that is based on numerous geographically and time-spread strains is characterised by a highly plastic and unusual fungal species that challenges this concept. In the majority of these $H$. werneckii strains, on the one hand, there were ambiguous positions for the household genes routinely used in taxonomic studies, and on the other hand, there were the unambiguous sequences of the ribosomal genes. According to our estimates, around $20 \%$ of these $H$. werneckii strains were haploid, while the others were diploid. This situation prevented standard species delineation and requires a restrained and adjusted taxonomic approach. Analysis of a smaller number of geographically separated strains or a restricted number of phenotypic and clustering analyses might have resulted in the description of several new species. We believe that 
whenever there is the need to deal with extremotolerant and (or) phenotypically variable fungi, as exemplified by many black yeasts, a tailor-made rather than a standard approach to species recognition should be considered.

\section{Additional file}

Additional file 1: Table S1. Genome mining for ITS and D1/D2 rDNA sequences. The number of reads with a sequence identical to the reference sequence of the strain EXF-225 (ref.) and number of reads with an alternative sequence (alt) are shown. Table S2. Halotolerance of the Hortaea werneckii strains over 9 weeks on malt extract agar without and with added 5, 10, 15, 20, 25, and 30\% NaCl. Table S3. Growth of the Hortaea werneckii at different temperatures $\left(5,15,25,37^{\circ} \mathrm{C}\right)$ on solid malt extract agar without and with added $10 \% \mathrm{NaCl}$. Table S4. Growth of the Hortaea werneckii strains on the different culture media. Table S5.

Conidia and hyphae measurements for the Hortaea werneckii strains after 2 weeks of incubation on oatmeal agar at $25^{\circ} \mathrm{C}$. Table S6. Carbon and nitrogen assimilation for the Hortaea werneckii strains. Table S7. Nitrogen assimilation for the Hortaea werneckii strains. Table S8. Proteolytic (casein, gelatine) and lipolytic (esterase) enzymes of the Hortaea werneckii strains grown on solid media at $25^{\circ} \mathrm{C}$ and $37^{\circ} \mathrm{C}$, and with addition of 0,5 , and $10 \% \mathrm{NaCl}$. (XLSX $64 \mathrm{~kb})$

\section{Abbreviations}

BTB: Partial genes encoding for $\beta$-tubulin; CBS: Acronym used for fungal strains preserved in Westerdijk Institute for Fungal Biodiversity Culture Collection, Utrecht, the Netherlands (former Centraalbureau voor Schimmelcultures); D1/D2 of 28S: D1/D2domains of 28S rDNA; EXF: Acronym used for fungal strains preserved in the Microbial Culture Collection (Ex) of the Infrastructural Centre Mycosmo, MRIC UL, Slovenia; ITS: Internal transcribed spacer of rDNA; MCM7: Partial genes encoding for minichromosome maintenance protein

\section{Acknowledgements}

We thank the Slovenian Research Agency for supporting the infrastructural centre Mycosmo MRIC UL and the Culture Collection of Extremophilic Fungi (Ex), Research Programmes P1-0170 and P1-0207, and Postdoctoral Project Z7-7436 to J. Zajc. We also thank the Ministry of Higher Education, Science and Technology of the Republic of Slovenia for the Young Researcher grant to J. Zupančič (grant no. 382228-1/2013). We would like to thank Chiara Alaimo for performing some of the enzymatic tests. Mojca Matul and Barbara Kastelic Bokal are acknowledged for their excellent technical assistance. A. A.B. thanks the European Research Council for starting grant 307496.

\section{Funding}

Slovenian Research Agency (Infrastructural centre Mycosmo MRIC UL, Research Programmes P1-0170 and P1-0207, and Postdoctoral Project Z77436 to J. Zajc). Ministry of Higher Education, Science and Technology of the Republic of Slovenia: Young Researcher grant to J.Zupančič (grant no. 382228-1/2013). European Research Council for starting grant 307496.

\section{Availability of data and materials}

All data generated or analysed during this study are included in this published article (and its supplementary information files).

\section{Adherence to national and international regulations}

We herewith confirm adhearance to national and international legislation.

\section{Authors' contributions}

Conceptualization, PZ \& NG-C; Methodology, PZ, JZupančič, JZajc, CG; Acquisition of, analysis and design of the work, PZ, CG, JZupančič, JZajc; Resources, PZ, FDL, AA-B, GSdH; Writing - Original Draft Preparation, PZ; Writing-Review \& Editing, CG, NG-C, SdH, FDL, AA-B; Acquisition, Analysis, Interpretation of data, PZ, JZupančič, JZajc, CG, NG-C. All authors read and approved the final manuscript.
Ethics approval and consent to participate

Not applicable.

\section{Consent for publication}

Not applicable.

\section{Competing interests}

The authors declare that they have no competing interests.

\section{Author details}

${ }^{1}$ Department of Biology, Biotechnical Faculty, University of Ljubljana, Večna pot 111, SI-1000 Ljubljana, Slovenia. ${ }^{2}$ Lars Bolund Institute of Regenerative Medicine, BGI-Qingdao, Qingdao 266555, China. ${ }^{3}$ Department of Biotechnology and Systems Biology, National Institute of Biology, Večna pot 111, 1000 Ljubljana, Slovenia. ${ }^{4}$ Westerdijk Fungal Biodiversity Institute, P.O. Box 85167, Utrecht 3508 AD, The Netherlands. ${ }^{5}$ Centre of Expertise in Mycology of RadboudUMC, Canisius Wilhelmina Hospital, Nijmegen, The Netherlands. ${ }^{6}$ Department of Chemical, Biological, Pharmaceutical and Environmental Sciences (ChiBioFarAm), University of Messina, Viale F. Stagno d'Alcontres, 3198166 Messina, Italy. ${ }^{7}$ Centro de Astrobiología (CSIC-INTA), Madrid, Spain. ${ }^{8}$ Instituto de Ciencias Biomédicas, Facultad de Ciencias de la Salud, Universidad Autónoma de Chile, 8910060 Santiago, Chile.

Received: 29 March 2019 Accepted: 3 April 2019

Published online: 08 July 2019

References

Alkan C, Coe BP, Eichler EE (2011) GATK toolkit. Nature Reviews Genetics 12:363376

Amend AS, Barshis DJ, Oliver TA (2012) Coral associated marine fungi form novel lineages and heterogeneous assemblages. The ISME Journal 6:1291-1301

Azúa-Bustos A, González-Silva C, Salas L, Palma RE, Vicuña R (2010) A novel subaerial Dunaliella species growing on cave spiderwebs in the Atacama Desert. Extremophiles 14:443-452

Bennett RJ, Johnson AD (2003) Completion of a parasexual cycle in Candida albicans by induced chromosome loss in tetraploid strains. The EMBO Journal 22:2505-2515

Boekhout T, Renting M, Scheffers WA, Bosboom R (1993) The use of karyotyping in the systematics of yeasts. Antonie Van Leeuwenhoek 63:57-63

Bonifaz A, Badali H, de Hoog GS, Araiza J, Cruz MA et al (2008) Tinea nigra by Hortaea werneckii, a report of 22 cases from Mexico. Studies in Mycology 61: 77-82

Brasier CM (2000) The rise of the hybrid fungi. Nature 405:134-135

Brauers G, Ebel R, Edrada RA, Wray V, Berg A et al (2001) Hortein, a new natural product from the fungus Hortaea werneckii associated with the sponge Aplysina aerophoba. Journal of Natural Products 64:651-652

Brizzio S, Turchetti B, de García V, Libkind D, Buzzini P et al (2007) Extracellular enzymatic activities of basidiomycetous yeasts isolated from glacial and subglacial waters of Northwest Patagonia (Argentina). Canadian Journal of Microbiology 53:519-525

Butinar L, Sonjak S, Zalar P, Plemenitaš A, Gunde-Cimerman N (2005) Melanized halophilic fungi are eukaryotic members of microbial communities in hypersaline waters of solar salterns. Botanica Marina 48:73-79

Cabañes FJ, Bragulat MR, Castellá G (2012) Hortaea werneckii isolated from silicone scuba diving equipment in Spain. Medical Mycology 50:852-857

Čadež N, Dlauchy D, Raspor P, Péter G (2013) Ogataea kolombanensis sp. nov., Ogataea histrianica sp. nov. and Ogataea deakii sp. nov., three novel yeast species from plant sources. International Journal of Systematic and Evolutionary Microbiology 63:3115-3123. https://doi.org/ 10.1099/ijs.0.052589-0

Cantrell SA, Casillas-Martínez L, Molina M (2006) Characterization of fungi from hypersaline environments of solar salterns using morphological and molecular techniques. Mycological Research 110:962-970

Chen J, Xing XK, Zhang LC, Xing YM, Guo SX (2012) Identification of Hortaea werneckii isolated from mangrove plant Aegiceras comiculatum based on morphology and rDNA sequences. Mycopathologia 174:457-466

Crous PW, Schoch CL, Hyde KD, Wood AR, Gueidan C et al (2009) Phylogenetic lineages in the Capnodiales. Studies in Mycology 64:17-47

Darriba D, Taboada GL, Doallo R, Posada D (2012) JModelTest 2: more models, new heuristics and parallel computing. Nature Methods 9:772 
de Hoog GS, Gerrits van den Ende AHG (1992) Nutritional pattern and ecophysiology of Hortaea werneckii, agent of human tinea nigra. Antonie Van Leeuwenhoek 62:321-329

de Hoog GS, Guarro J, Gené J, Figueras MJ (2000) Atlas of clinical fungi, 2nd edn. Centraalbureau voor Schimmelcultures/ Universitat Rovira i Virgili, Utrecht, pp $720-722$

de Hoog GS, Guého E (1998) Agents of white piedra, black piedra and tinea nigra. In: Ajello L, Hay RJ (eds) Topley \& Wilson's microbiology and microbial infections, vol 4, 9th edn. Arnold, London, pp 191-197

de Hoog GS, Zalar P, Urzi C, De Leo F, Yurlova NA et al (1999) Relationships of dothideaceous black yeasts and meristematic fungi based on 5.85 and ITS2 rDNA sequence comparison. Studies in Mycology 43:31-37.

de Hoog GS, Zalar P, van den Ende BG, Gunde-Cimerman N (2005) Relation of halotolerance to human-pathogenicity in the fungal tree of life: an overview of ecology and evolution under stress. In: Adaptation to life at high salt concentrations in Archaea, Bacteria, and Eukarya, (Cellular origin, life in extreme habitats and astrobiology, 9). (N Gunde-Cimerman, A Oren, A Plemenitaš, eds): $371-395$. Springer, the Netherlands

De Leo F, Giudice AL, Alaimo C, De Carlo G, Rappazzo AC et al (2018) Occurrence of the black yeast Hortaea werneckii in the Mediterranean Sea. Extremophiles https://doi.org/10.1007/s00792-018-1056-1

Díaz-Muñoz G, Montalvo-Rodríguez R (2005) Halophilic black yeast Hortaea werneckii in the Cabo Rojo solar salterns: its first record for this extreme environment in Puerto Rico. Caribbean Journal of Science 41: 360-365

Elsayed A, Mowafy M, Soliman HM, Gebreil A, Magdy NI (2016) Characterization of new strains of Hortaea werneckii isolated from salt marshes of Egypt. Egyptian Journal of Basic and Applied Sciences 3:350-356

Fogelqvist J, Tzelepis G, Bejai S, Ilbäck J, Schwelm A et al (2018) Analysis of the hybrid genomes of two field isolates of the soil-borne fungal species Verticillium longisporum. BMC Genomics 19:14. https://doi.org/10.1186/ s12864-017-4407-x

Formoso A, Heidrich D, Felix CR, Tenório AC, Leite BR et al (2015) Enzymatic activity and susceptibility to antifungal agents of brazilian environmental isolates of Hortaea werneckii. Mycopathologia 180:345-352

Gerrits van den Ende AHG, de Hoog GS (1999) Variability and molecular diagnostics of the neurotropic species Cladophialophora bantiana. Studies in Mycology 43:151-162

Glass NL, Donaldson GC (1995) Development of primer sets designed for use with the PCR to amplify conserved genes from filamentous ascomycetes. Applied and Environmental Microbiology 61:1323-1330

Gostinčar C, Lenassi M, Gunde-Cimerman N, Plemenitaš A (2011) Fungal adaptation to extremely high salt concentrations. Advances in Applied Microbiology 77:71-96

Gostinčar C, Stajich JE, Zupančič J, Zalar P, Gunde-Cimerman N (2018) Genomic evidence for intraspecific hybridization in a clonal and extremely halotolerant yeast. BMC Genomics 19:364 https://doi.org/10.1186/s12864-018-4751-5

Guindon S, Dufayard JF, Lefort V, Anisimova M, Hordijk W et al (2010) New algorithms and methods to estimate maximum-likelihood phylogenies: assessing the performance of PhyML 3.0. Systematic Biology 59:307-321

Gunde-Cimerman N, Plemenitaš A, Oren A (2018) Strategies of adaptation of microorganisms of the three domains of life to high salt concentrations. FEMS Microbiology Reviews 42:353-375 https://doi.org/10.1093/femsre/fuy009

Gunde-Cimerman N, Sonjak S, Zalar P, Frisvad JC, Diderichsen B et al (2003) Extremophilic fungi in arctic ice: a relationship between adaptation to low temperature and water activity. Physics and Chemistry of the Earth 28:1273-1278

Gunde-Cimerman N, Zalar P (2014) Extremely halotolerant and halophilic fungi inhabit brine in solar salterns around the globe. Food Technology and Biotechnology 52:170-179

Gunde-Cimerman N, Zalar P, de Hoog GS, Plemenitaš A (2000) Hypersaline water in salterns and natural ecological niches for halophilic black yeast. FEMS Microbiology Ecology 32:235-240

Hammer $\varnothing$, Harper DAT, Ryan PD (2001) PAST: Palaeontological statistics software package for education and data analysis. Palaeontologia Electronica 4(1):9

Hankin L, Anagnostakis SL (1975) Use of solid media for detection of enzyme production by fungi. Mycologia 67:597-607. https://doi.org/10.2307/3758395

Hölker U, Bend J, Pracht R, Tetsch L, Müller T et al (2004) Hortaea acidophila, a new acid-tolerant black yeast from lignite. Antonie Van Leeuwenhoek 86: 287-294
Hujslova M, Kubatova A, Chudickova M, Kolarik M (2010) Diversity of fungal communities in saline and acidic soils in the Soos National Natural Reserve, Czech Republic. Mycological Progress 9:1-5

Humphries Z, Seifert KA, Hirooka Y, Visagie CM (2017) A new family and genus in Dothideales for Aureobasidium-like species isolated from house dust. IMA Fungus 8:299-315

Inderbitzin P, Bostock RM, Davis RM, Usami T, Platt HW et al (2011a) Phylogenetics and taxonomy of the fungal vascular wilt pathogen Verticillium, with the descriptions of five new species. PLoS One 6:e28341. https://doi.org/10.1371/journal.pone.0028341

Inderbitzin P, Davis RM, Bostock RM, Subbarao KV (2011b) The ascomycete Verticillium longisporum is a hybrid and a plant pathogen with an expanded host range. PLoS One 6:e18260. https://doi.org/10.1371/journal. pone.0018260

Iwatsu TU, Udagawa S (1988) Hortaea werneckii isolated from sea-water. Japanese Journal of Medical Mycology 29:142-145

Kamvar ZN, Brooks JC, Grünwald NJ (2015) Novel R tools for analysis of genomewide population genetic data with emphasis on clonality. Frontiers in Genetics 6. https://doi.org/10.3389/fgene.2015.00208

Katoh K, Toh H (2008) Recent developments in the MAFFT multiple sequence alignment program. Briefings in Bioinformatics 9:286-298

Kogej T, Stein M, Volkmann M, Gorbushina AA, Galinski EA et al (2007) Osmotic adaptation of the halophilic fungus Hortaea werneckii: role of osmolytes and malanization. Microbiology 153:4261-4273

Kornerup A, Wanscher JH (1978) 12th Methuen handbook of colour, 3rd edn. Methuen, UK

Lai X, Cao L, Tan H, Fang S, Huang Y, Zhou S (2007) Fungal communities from methane hydrate-bearing deep-sea marine sediments in South China Sea. The ISME Journal 1:756-762

Lenassi M1, Gostinčar C, Jackman S, Turk M, Sadowski I et al (2013) Whole genome duplication and enrichment of metal cation transporters revealed by de-novo genome sequencing of extremely halotolerant black yeast Hortaea werneckii. PLoS One 8:e71328. https://doi.org/10.1371/journal.pone. 0071328 eCollection 2013

Li H, Durbin R (2009) Fast and accurate short read alignment with burrowswheeler transform. Bioinformatics 25:1754-1760

Li H, Handsaker B, Wysoker A, Fennell T, Ruan J et al (2009) The sequence alignment/ map format and SAMtools. Bioinformatics 25:2078-2079

Liao D (1999) Concerted evolution: molecular mechanism and biological implications. American Journal of Human Genetics 64:24-30

Luo ZH, Xu WE, Guo S-S (2017) Fungal diversity in deep sea floor of the Pacific Ocean, 2nd International Conference of Marine Fungal Natural Products (MaFNaP_2017), 27-29 June, Kiel, p 49

Marcet-Houben M, Gabaldón T (2015) Beyond the whole-genome duplication: phylogenetic evidence for an ancient interspecies hybridization in the baker's yeast lineage. PLoS Biology 13(8):e1002220 https://doi.org/10.1371/ journal.pbio.1002220

Marchetta A, Gerrits van den Ende B, Al-Hatmi AMS, Hagen F, Zalar P et al (2018) Global molecular diversity of the halotolerant fungus Hortaea werneckii. Life 8:31. https://doi.org/10.3390/life8030031

Martinelli L, Zalar P, Gunde-Cimerman N, Azua-Bustos A, Sterfinger K et al (2017) Aspergillus atacamensis and A. salisburgensis: two new halophilic species from hypersaline/ arid habitats with a phialosimplex-like morphology. Extremophiles 21:755-773

McManus BA, Coleman DC (2014) Molecular epidemiology, phylogeny and evolution of Candida albicans. Infection, Genetics and Evolution 21:166-178

Mok WY, Castello FP, Baretto de Silva MS (1981) Occurence of Exophiala werneckil on salted freshwater fish Osteloglossum bicirrhosum. Journal of Food Science and Technology 16:505-512

Nai C, Wong HY, Pannenbecker A, Broughton WJ, Benoit I et al (2013) Nutritional physiology of a rock-inhabiting, model microcolonial fungus from an ancestral lineage of the Chaetothyriales (ascomycetes). Fungal Genetics and Biology 56:54-66

Naidoo K, Steenkamp ET, Coetzee MPA, Wingfield MJ, Wingfield BD (2013) Concerted evolution in the ribosomal RNA cistron. PLoS One 8. https://doi. org/10.1371/journal.pone.0059355

Ng KP, Soo-Hoo TS, Na SL, Tay ST, Hamimah H et al (2005) The mycological and molecular study of Hortaea werneckii isolated from blood and splenic abscess. Mycopathologia 159:495-500

Nishimura K, Miyaji M (1984) Hortaea, a new genus to accomodate Cladosporium werneckii. Japanese Journal of Medical Mycology 25:139-146 
O'Donnell K (2000) Molecular phylogeny of the Nectria haemato-cocca-Fusarium solani species complex. Mycologia 92:919-938

Ortiz-Merino RA, Kuanyshev N, Braun-Galleani S, Byrne KP, Porro D et al (2017) Evolutionary restoration of fertility in an interspecies hybrid yeast, by wholegenome duplication after a failed mating-type switch. PLoS Biology 15 e2002128 https://doi.org/10.1371/journal.pbio.2002128

Paneque Rodríguez I, León García Y, Vidal Rosell D, Guedes Vidal L, IIInait Zaragozí MT et al (2015) Reports of seven cases of tinea nigra in children/ Presentación de siete casos de tiña negra en niños (in Spanish). Revista Cubana de Medicina Tropical 67(3) https://www.medigraphic.com/cgi-bin/ new/resumenl.cgi?IDARTICULO=66437

Paterson RRM, Bridge PD (1994) Biochemical techniques for filamentous fungi. IMI Technical Habdbooks, CAB International, UK

Perez C, Colella MT, Olaizola C, de Capriles CH, Magaldi S et al (2005) Tinea nigra: report of twelve cases in Venezuela. Mycopathologia 160:235-238

Plemenitaš A, Gunde-Cimerman N (2005) Cellular reponses in the halophilic black yeast Hortaea werneckii to high environmental salinity. In: Gunde-Cimerman N, Oren A, Plemenitaš A (eds) Adaptation to life at high salt concentrations in Archea, Bacteria and Eukarya. Springer, The Netherlands, pp 455-470

Plemenitaš A, Lenassi M, Konte T, Kejžar A, Zajc J et al (2014) Adaptation to high salt concentrations in halotolerant/ halophilic fungi: a molecular perspective. Frontiers in Microbiology 5. https://doi.org/10.3389/fmicb.2014.00199

Plemenitaš A, Vaupotič T, Lenassi M, Kogej T, Gunde-Cimerman N (2008) Adaptation of extremely halotolerant black yeast Hortaea werneckii to increased osmolarity: a molecular perspective at a glance. Studies in Mycology 61:67-75

Quaedvlieg W, Binder M, Groenewald JZ, Summerell BA, Carnegie AJ et al (2014) Introducing the consolidated Sspecies concept to resolve species in the Teratosphaeriaceae. Persoonia 33:1-40

R Development Core Team (2017) R: A language and environment for statistical computing.

Rossetto AL, Corrêa PR, Cruz RCB, Pereira EF, Haddad-Júnior V (2014) A case of tinea nigra associated to a bite from a European rabbit (Oryctolagus cuniculus, Leporidae): the role of dermoscopy in diagnosis. Anais Brasileiros de Dermatologia 89:165-166

Ruibal C, Gueidan C, Selbmann L, Gorbushina AA, Crous PW et al (2009) Phylogeny of rock-inhabiting fungi related to Dothideomycetes. Studies in Mycology 64:123-133

Schliep K, Potts AJ, Morrison DA, Grimm GW (2017) Intertwining phylogenetic trees and networks. Methods in Ecology and Evolution 8:1212-1220

Schmitt I, Crespo A, Divakar PK, Fankhauser JD, Herman-Sackett E et al (2009) New primers for promising single-copy genes in fungal phylogenetics and systematic. Persoonia 23:35-40

Selbmann L, de Hoog GS, Mazzaglia A, Friedmann El, Onofri S (2005) Fungi at the edge of life: cryptoendolithic black fungi from Antarctic deserts. Studies in Mycology 51:1-32

Singh P, Raghukumar C, Meena RM, Verma P, Shouche Y (2012) Fungal diversity in deep-sea sediments revealed by culture-dependent and cultureindependent approaches. Fungal Ecology 5:543-553

Sinha S, Flibotte S, Neira M, Formby S, Plemenitaš A et al (2017) Insight into the recent genome duplication of the halophilic yeast Hortaea werneckii: combining an improved genome with gene expression and chromatin structure. G3 (Bethesda) 7(7):2015-2022

Smedile F, Scarfi S, De Domenico E, Garel MH, Gentile G et al (2015) Variations in microbial community structure through the stratified water column in the Tyrrhenian Sea (Central Mediterranean). Marine Science and Engineering 3:845-865

Stephens M, Smith NJ, Donnelly P (2001) A new statistical method for haplotype reconstruction from population data. American Journal of Human Genetics 68:978-989

Strauss ML, Jolly NP, Lambrechts MG, van Rensburg P (2001) Screening for the production of extracellular hydrolytic enzymes by non Saccharomyces wine yeasts. Journal of Applied Microbiology 91:182-190

Sunshine AB, Payen C, Ong GT, Liachko I, Ming Tan K et al (2015) The fitness consequences of aneuploidy are driven by condition-dependent gene effects. PLoS Biology 13:e1002155

Teixeira MM, Moreno LF, Stielow BJ, Muszewska A, Hainaut M et al (2017) Exploring the genomic diversity of black yeasts and relatives (Chaetothyriales, Ascomycota). Studies in Mycology 86:1-28. https://doi.org/10.1016/j.simyco. 2017.01.001

Todaro F, Berdar A, Cavaliere A, Criseo G, Pernice L (1983) Gasophthalmus in black sea bream (Spondyliosoma cantharus) caused by Sarcinomyces crustaceous Lindner. Mycopathologia 81:95-97
Uijthof JM, de Cock AW, de Hoog GS, Quint WG, van Belkum A (1994) Polymerase chain reaction-mediated genotyping of Hortaea werneckii, causative agent of tinea nigra. Mycoses 37:307-312

Vaupotič T, Plemenitaš A (2007) Osmoadaptation-dependent activity of microsomal $\mathrm{HMG}-\mathrm{CoA}$ reductase in the extremely halotolerant black yeast Hortaea werneckii is regulated by ubiquitination. FEBS Letters 581: 3391-3395

Vaupotič T, Veranič P, Petrovič U, Gunde-Cimerman N, Plemenitaš A (2008) HMG$\mathrm{CoA}$ reductase is regulated by environmental salinity and its activity is essential for halotolerance in halophilic fungi. Studies in Mycology 61:61-66. https://doi.org/10.3114/sim.2008.61.05

Viti C, Decorosi F, Marchi E, Galardini M, Giovannetti L (2015) High-throughput phenomics. In: Mengoni A, Galardini M, Fondi M (eds) Bacterial pangenomics: methods and protocols. Springer, USA, pp 99-123

White TJ, Bruns T, Lee S, Taylor J (1990) Amplification and direct sequencing of fungal ribosomal RNA genes for phylogenetics. In: PCR protocols: a guide to methods and applications. Academic Press, USA

Wollenzien U, de Hoog GS, Krumbein WE, Urzi C (1995) On the isolation of microcolonial fungi occuring on and in marble and other calcareous rocks. Science of the Total Environment 167:287-294

Xu W, Pang K-L, Luo Z-H (2014) High fungal diversity and abundance recovered in the deep-sea sediments of the Pacific Ocean. Microbial Ecology 68:688-698

Zajc J, Džeroski S, Kocev D, Oren A, Sonjak S et al (2014) Chaophilic or chaotolerant fungi: a new category of extremophiles? Frontiers in Microbiology 5:708. https://doi.org/10.3389/fmicb.2014.00708

Zalar P, de Hoog GS, Gunde-Cimerman N (1999) Ecology of halotolerant dothideaceous black yeasts. Studies in Mycology 43:38-48

Zhang X-Y, Tang G-L, Xu X-Y, Nong X-H, Qi S-H (2014) Insights into deep-sea sediment fungal communities from the East Indian Ocean using targeted environmental sequencing combined with traditional cultivation. PLoS One 9(10):e109118. https://doi.org/10.1371/journal.pone.0109118

\section{Publisher's Note}

Springer Nature remains neutral with regard to jurisdictional claims in published maps and institutional affiliations.

\section{Ready to submit your research? Choose BMC and benefit from:}

- fast, convenient online submission

- thorough peer review by experienced researchers in your field

- rapid publication on acceptance

- support for research data, including large and complex data types

- gold Open Access which fosters wider collaboration and increased citations

- maximum visibility for your research: over $100 \mathrm{M}$ website views per year

At BMC, research is always in progress.

Learn more biomedcentral.com/submissions 